\title{
Adaptive Observers With Persistency of Excitation for Synchronization of Chaotic Systems
}

\author{
Antonio Loría, Member, IEEE, Elena Panteley, and Arturo Zavala-Río
}

\begin{abstract}
We address the problem of master-slave synchronization of chaotic systems under parameter uncertainty and with partial measurements. Our approach is based on observer-design theory hence, we view the master dynamics as a system of differential equations with a state and a measurable output and we design an observer (tantamount to the slave system) which reconstructs the dynamic behavior of the master. The main technical condition that we impose is persistency of excitation (PE), a property well studied in the adaptive control literature. In the case of unknown parameters and partial measurements we show that synchronization is achievable in a practical sense, that is, with "small" error. We also illustrate our methods on particular examples of chaotic oscillators such as the Lorenz and the Lü oscillators. Theoretical proofs are provided based on recent results on stability theory for time-varying systems.
\end{abstract}

Index Terms-Adaptive control, chaos control, chaotic systems, observers, synchronization.

\section{INTRODUCTION}

\section{A. On Controlled Synchronization}

$\mathbf{S}$ INCE the seminal work of Blekhman [1] systems synchronization has attracted growing attention in different scientific communities, ranging from that of physics, electrical engineering, control theory, signal processing, to mention a few. Synchronization appears in different ways and circumstances but mainly in the so-called master-slave configuration, in which a leader system marks the pace to a follower system, and $m u$ tual in which generally more than two systems synchronize their motion with respect to each other without any hierarchy.

We focus on the problem of master-slave synchronization of chaotic systems. This was mainly initiated by the celebrated paper of Pecora and Carroll [2] and has triggered a number of works in the subject, motivated by applications such as (but not exclusively) encoding of information for secure transmission—cf. e.g., [3]-[9]. During the last 15 years or so, a number of methods to establish synchronization of chaotic systems have been proposed in more or less generality: for instance, focussed on the Lorenz system: [5], [10], [11] (one of the most popular chaotic oscillators) or covering relatively general classes of chaotic oscillators—cf. [6], [7], [12]-[16].

Manuscript received June 13, 2008; revised December 19, 2008. First published March 06, 2009; current version published January 13, 2010. This paper was recommended by Associate Editor O. De Feo.

A. Loría and E. Panteley are with French National Centre of Scientific Research (CNRS), Laboratoire de Signaux et Systemes (LSS-SUPELEC), 91192 Gif s/Yvette, France (e-mail: loria@1ss.supelec.fr).

A. Zavala-Río is with the Instituto Potosino de Investigación Científica y Tecnológica, 78216 San Luis Potosí, Mexico (e-mail: azavala@ipicyt.edu.mx).

Digital Object Identifier 10.1109/TCSI.2009.2016636
Beyond those of Physics and Electrical Engineering, viewpoints that have proved to be useful to synchronization are that from control theory, for design methods, and that from stability theory, for analysis. Indeed, two general questions that are recurrently investigated in synchronization of chaotic systems are: 1) under which conditions systems synchronize (values of physical parameters, initial conditions, etc.) and consequently, 2) under which conditions one is able to design chaotic systems that synchronize with others? To answer the first question Lyapunov stability theory may be used — $c$. [17]-[19], for the second, distinct control approaches have been put to test-cf. [6], [7], [12]-[14]. Some papers rely on analytic study -[8], $[20],[21]$ and others on numerical methods and validation in simulation —cf. [16], [22], [23]. Beyond stability theory, work on synchronization analysis includes the study of synchronization in networks of oscillators - cf. [24]-[29], relying on tools from e.g., graph theory. Fundamental work on pinning-controllability just appeared in [24]. In [26] conditions are established in terms of the average coupling path lengths among network nodes; see also [25]. The recent paper [28] establishes synchronizability in terms of conditions on parameters of the probability distribution that governs the topological changes of the network. A number of articles establish fast-switching conditions relying on averaging techniques (also well-known in the theory of systems stability). In the recent paper [27] it is established that synchronization may occur even if connections are instantaneously lost 'at times' as long as the interconnection among all nodes is kept in average. Other recent articles relying on fast-switching conditions include [30] which deals with stochastic models i.e., the nodes' couplings switch on and off randomly.

The method presented in [15] which may also be re-casted in the context of identification plus synchronization relies on synchronizing the master and slave systems under parameter uncertainty at the expense of synchronization mismatch then, an adaptive algorithm is activated locally to estimate the parameters. The article [16] has triggered many other works on adaptive synchronization and identification of chaotic systems. However, as pointed out for instance in [17], [31] the method from [16] does not work in general: in [31] an alternative proof for parametric convergence is given which relies on La Salle's invariance principle or, a variant of it seemingly for chaotic systems ${ }^{1}$. In [17] a proof of convergence of synchronization errors is established following "signal-chasing" arguments standard in adaptive control theory but parametric convergence is not established, it is only observed (for the particular case of the Lorenz system) that parameters converge when the system is in a chaotic or in a periodic regime -this is stressed as an "inter-

\footnotetext{
${ }^{1}$ While we are not aware of a precise formulation of La Salle's invariance principle for chaotic systems we stress that this theorem does not apply in general to time-varying systems (except periodic).
} 
esting phenomenon which remains to be further investigated". This phenomenon has been studied and explained recently in [32] in terms of so-called persistency of excitation (PE). As a matter of fact, this notion is known in adaptive control literature for about 40 years now and, within a specific setting ${ }^{2}$, it is a necessary condition for parametric convergence. It also seems closely related to the averaging techniques used e.g., in [25], [26] and [27].

Among the many control-theory based approaches to synchronization we single out that based on observer theory (Luenberger, high-gain, adaptive, reduced-order, etc.). An observer is, roughly speaking, a dynamical system designed to estimate the states of another. As it has been laid out in [35] master-slave synchronization a la Pecora-Carroll may be recasted in the context of observer design. This is significant as observer theory is well-developed, mainly for linear systems — $c f$. [36], [37] but also with important advancements for nonlinear systems- $c f$. [38]. Within the vast observer theory we shall emphasize adaptive observer design. In this case, it is required to design an observer under parametric uncertainty. In the context of synchronization of chaotic systems this translates into the problem of designing a slave system which (as an observer) tracks (that is, estimates) the trajectories of a master system by measuring a function or part of the master's state. This is in high contrast with many of the previous references where it is assumed that a control action may be exerted by, possibly, measuring the whole state-cf. e.g., [20], [39]-[42]. Early work on observer-based synchronization includes [43]; more recent work, including parametric uncertainty and adaptation are the interesting papers [12], [44] of which we became aware after the original submission of this paper. In the first reference the authors present similar results to ours but under somewhat more restrictive assumptions: adaptive observers for partially linear systems, affine in the unmeasured variables and, under persistency of excitation conditions, conclude synchronization and parametric convergence with small errors. The second reference is targeted to applications in the realm of secured communication based on synchronization of chaotic systems.

Generically, adaptive synchronization with full measurement of the master system is an important and challenging problem in its own right. In this context it is worth mentioning the interesting and efficient open-loop identification scheme of [45] but which is limited to systems having one unknown parameter per dynamic equation and with a specific structure. Closed-loop identification may be recasted in the context of so called adaptive tracking control for chaotic systems-cf. [32], [39], [46]. Roughly observer-based synchronization, which is the subject of study in this paper, is comparable to a problem of tracking control with partially unknown reference operating regime.

Literature on observer theory is very rich and we shall not deal with a review; we only mention a few papers related to the method we employ here. In that respect, let us recall the articles [47]-[50] whose formulations are reminiscent of the early works [51], [52]. The papers [47]-[49] deal with linear time-varying systems and both parametric and state estimation errors are guaranteed to converge under a condition formulated

\footnotetext{
${ }^{2}$ By "specific setting" we refer to classes of systems having certain structural properties related, for instance, to relative degree; so-called Model Reference Adaptive Control systems, etc. We shall not describe these in detail, readers are kindly suggested to see [33], [34].
}

in terms of persistency of excitation. The article [50], among others by the same author, deals with a similar problem for a class of nonlinear systems which has two particularly important features: the system is linear in the unknown variables (unmeasured states and parameters) and, moreover, parametric uncertainty appears only in the set of dynamic equations of the measured variables. Strictly speaking, the result is stated for such class of systems but holds for more general forms provided a coordinate transformation exists which brings a "general" nonlinear system into the desired form. More on such coordinate transformations can be found for instance in [53], [54] to cite a few. Recent articles on adaptive observers for systems, linear in the unmeasured states specifically with the purpose of addressing synchronization of chaotic systems include [6]-[8], [12], [14], [35], [55]-[58]; in many of these references (at least in [8], [55]-[57]) stringent conditions such as (global) Lipschitz on the nonlinearities are assumed to hold.

Other techniques for controlled synchronization rely on timevarying delay control. In particular, some results for systems of the Lur'e type-cf.e.g., [59] that is, with sector nonlinearities (hence a similar class of systems covered by observer theory as previously discussed) include [60], [61]. The last three references have also appeared during the review process of this paper.

\section{B. About This Paper}

In this article we present sufficient conditions for parametric and state estimation using adaptive observers which cover highgain designs_ $c f$. [35], [51] and others from the references cited above: 1) we lay sufficient general conditions in terms of persistency of excitation along trajectories for nonlinear systems (in contrast to [47]-[49]); 2) the class of nonlinear systems includes systems that are linear in the unknown variables but parametric uncertainty may appear anywhere in the model (in contrast to [50]); 3) the conditions we set for our adaptive observers intersect (and generalize in certain ways) with high-gain designs for nonlinear systems, similar to those in [62], [63] however, in contrast to the former our method is not restricted to high-gain observers and, with respect to the latter, in this paper we cover the case of parametric uncertainty without controls; 4) the class of systems that we consider contains time-varying nonlinearities which may be regarded as neglected dynamics; in contrast with works relying on Lipschitz assumptions we allow for high order terms provided that the trajectories of the master system are bounded (which is not restrictive in the context of chaotic systems); 5) the condition in terms of PE covers cases considered for instance in [7], [8], [12], [47]-[49], [55]-[58]. Our results are for a (structurally) similar class of systems as that considered in [12] except that in the latter systems are assumed to be partially linear; another fundamental difference is that in [12] synchronization is considered as making two respective outputs which are part of the state of the master and slave system converge to each other, as opposed to estimating the whole state of the system. The theoretical proofs that we present are original and rely on previous results for stability of parameterized linear systems-cf. [64].

In our main theorems we state and prove that under relaxed assumptions (in terms of persistency of excitation and structural conditions such as detectability and observability) the synchronization and parametric errors converge to compact sets, that is, 
the errors are bounded and relatively small (this is called practical asymptotic stability). Under more stringent conditions, for instance, in the case that parameters are known but not the states, synchronization may be achieved. Similarly, the adaptive observers may be employed into the specific problem of parameter identification if full measurement of master states is available. This situation is similar to the context of tracking control with full state-feedback as mentioned earlier.

The rest of the paper is organized as follows. In Section II we introduce some notation and definitions of stability that set the framework for our main results. These are presented in an increasing level of generality, accompanied with case-studies. In Section III we present the simplest case, that is, when all parameters are known and only partial measurements of the master system are available; in Section IV we study the case when the constant lumped parameters are unknown and we add an adaptation algorithm to estimate them; in Section V we present the most general result, for system's synchronization with partial measurement, parameter uncertainties and additional nonlinearities which may correspond to neglected dynamics or undesirable external disturbances acting on the system. Along each of the preceding sections we present case-studies and simulation results that illustrate our findings. All the proofs of stability are presented in an appendix at the end of the paper. We conclude with some remarks in Section VI.

\section{PRELIMINARIES}

Notation. We say that a function $\phi: \mathbb{R}_{\geq 0} \times \mathbb{R}^{n} \rightarrow \mathcal{A}$ where $\mathcal{A}$ is a closed, not necessarily compact set, satisfies the basic regularity assumption (BRA) if $\phi(t, \cdot)$ is locally Lipschitz uniformly in $t$ and $\phi(\cdot, x)$ is measurable. We denote the usual Euclidean norm of vectors by $\|\cdot\|$ and use the same symbol for the matrix induced norm. A function $\alpha: \mathbb{R}_{>0} \rightarrow \mathbb{R}_{>0}$ is said to be of class $\mathcal{K}(\alpha \in \mathcal{K})$, if it is continuous, strictly increasing and equals to zero at zero; $\alpha \in \mathcal{K}_{\infty}$ if, in addition, it is unbounded. A function $\beta: \mathbb{R}_{\geq 0} \times \mathbb{R}_{\geq 0} \rightarrow \mathbb{R}_{\geq 0}$ is of class $\mathcal{K} \mathcal{L}$ if $\beta(\cdot, t) \in \mathcal{K}, \beta(s, \cdot)$ is strictly decreasing and $\lim _{t \rightarrow \infty} \beta(s, t)=0$. We denote the solution of a differential equation $\dot{x}=f(t, x)$ starting at $x_{\mathrm{O}}$ at time $t_{\circ}$ by $x\left(\cdot, t_{\circ}, x_{\circ}\right)$; furthermore, if the latter are defined for all $t \geq t_{\circ}$ we say that the system is forward complete.

Definition 1 (Uniform Global Stability): The origin of

$$
\dot{x}=f(t, x)
$$

where $f(\cdot, \cdot)$ satisfies the BRA, is said to be uniformly globally stable (UGS) if there exists $\kappa \in \mathcal{K}_{\infty}$ such that, for each $\left(t_{\circ}, x_{\circ}\right) \in \mathbb{R}_{\geq 0} \times \mathbb{R}^{n}$, each solution $x\left(\cdot, t_{\circ}, x_{\circ}\right)$ of (1) satisfies

$$
\left\|x\left(t, t_{\circ}, x_{\circ}\right)\right\| \leq \kappa\left(\left\|x_{\circ}\right\|\right) \quad \forall t \geq t_{\circ} .
$$

Definition 2 (Uniform Global Asymptotic Stability): The origin of (1) is said to be uniformly globally asymptotically stable (UGAS) if it is UGS and uniformly globally attractive i.e., for each pair of strictly positive real numbers $(r, \sigma)$, there exists $T>0$ such that

$$
\left\|x_{\circ}\right\| \leq r \Longrightarrow\left\|x\left(t, t_{\circ}, x_{\circ}\right)\right\| \leq \sigma \quad \forall t \geq t_{\circ}+T .
$$

Definition 3 (Uniform Exponential Stability): The origin of the system $\dot{x}=f(t, x)$ is said to be uniformly exponentially stable on any ball if for any $r>0$ there exist two constants $k$ and $\gamma>0$ such that, for all $t \geq t_{\circ} \geq 0$ and all $x_{\circ} \in \mathbb{R}^{n}$ such that $\left\|x_{\circ}\right\|<r$ we have

$$
\left\|x\left(t, t_{\circ}, x_{\circ}\right)\right\| \leq k\left\|x_{\circ}\right\| e^{-\gamma\left(t-t_{\circ}\right)} .
$$

Definition 4 (Unif. Semiglobal Practical Asympt. Stability): The origin of (1) is said to be uniformly semi-globally practically asymptotically stable (USPAS) if for each positive real numbers $\Delta>\delta>0$ and $\sigma>0$ there exist $T>0$ and $\kappa \in \mathcal{K}_{\infty}$ such that $\left\|x\left(t, t_{\circ}, x_{\circ}\right)\right\| \leq \kappa\left(\left\|x_{\circ}\right\|\right)$ for all $t \geq t_{\circ} \geq 0$ and

$$
\left\|x_{\circ}\right\| \leq \Delta \Longrightarrow\left\|x\left(t, t_{\circ}, x_{\circ}\right)\right\| \leq \sigma+\delta \quad \forall t \geq t_{\circ}+T .
$$

For Definitions 1-3, for Definition 3 see [66]; for Definition 4 see [67].

\section{SYNCHRONIZATION AND OBSERVERS With PERSISTENCY OF EXCITATION}

\section{A. On Observability and Persistency of Excitation}

Consider a nonlinear system of the form

$$
\dot{x}=A(y) x
$$

where $x \in \mathbb{R}^{n}$ is the state vector; $y=C x, y \in \mathbb{R}^{m}$, is a measurable output. As explained in [35] synchronization a la Pecora and Carroll may be recasted in the context of observer design. Generally speaking, an observer system for (4) is a system with state $\hat{x}$ such that $\|\hat{x}(t)-x(t)\| \rightarrow 0$ as $t \rightarrow \infty$. In the context of master-slave synchronization, $\hat{x}$ may be thought of as the state variable of the slave system. Thus, one may solve the master-slave synchronization problem if one can design an observer for (4).

In the literature of nonlinear observers (cf. [35], [38]) a standard assumption is that the pair $(C, A(y))$ is $o b$ servable from the output $y$. To explain this condition let us first consider the case when $A$ is constant; we say that $(C, A)$ is observable if and only if the matrix

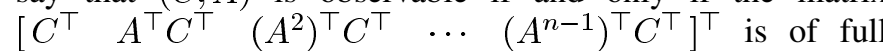
column rank. We stress that, in the case that $A$ is not constant, for instance, if $A$ is a function of $y$, one must require this condition to hold for each $y \in \mathbb{R}^{m}$. On occasions, one may ask the following less restrictive property: let $\left(t_{0}, x_{\circ}\right)$ with $x_{\circ}=x\left(t_{\circ}\right)$ be a pair of initial conditions that generate a trajectory (solution) of System (4) which we denote by $x\left(t ; t_{\circ}, x_{\circ}\right)$. In such case, the corresponding output trajectory is $y\left(t ; t_{\circ}, x_{\circ}\right)=C x\left(t ; t_{\circ}, x_{\circ}\right)$ or, in short, $y(t)=C x(t)$. For this particular output trajectory, generated by the particular pair of initial conditions $t_{\circ}$ and $x\left(t_{\circ}\right)$, the observability

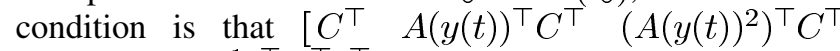
$\left.\cdots\left(A(y(t))^{n-1}\right)^{\top} C^{\top}\right]^{\top}$ has full column rank $n$ for each $t \geq t_{0}$. Notice that this is less restrictive than requiring that

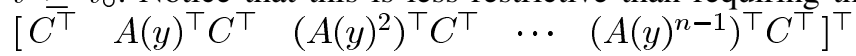
is of full column rank for all $y \in \mathbb{R}^{m}$. This is clear if we consider that the set of points $y(t)$ for all $t \geq t_{\circ}$ is for a particular pair of initial conditions, only a subset of $\mathbb{R}^{m}$. Hence, requiring observability for all $y \in \mathbb{R}^{m}$ is tantamount to requiring that the system is observable for all trajectories generated by any pair of initial conditions $t_{\circ} \geq 0, x_{\circ} \in \mathbb{R}^{n}$.

Another useful concept from control theory, in the design of observers and therefore, to solve the problem of synchronization 
a la Pecora and Carroll is detectability. Roughly, we require that the column rank of the matrix at the bottom of the page be full for some $T>0$ and for all $t \geq 0$. That is, it is no longer required that the rank condition holds for each $t$ but over a window of length $T$. For instance the matrix

$$
R(t):=\left[\begin{array}{cc}
|\sin (t)| & 0 \\
0 & |\cos (t)|
\end{array}\right]
$$

looses rank at $t \in\{0, n \pi / 2\}$ for all integer values of $n$ however, its integral over a window $[t, t+\pi / 2]$ is of rank 2 for any value of $t$.

In the literature of control systems and identification there exists a well known concept that is instrumental in understanding and stating conditions for detectability and observability of dynamical systems. Such property is known as persistency of excitation and its precise definition is as follows.

Definition 5 (Persistency of Excitation): A continuous ${ }^{3}$ function $\phi: \mathbb{R}_{\geq 0} \rightarrow \mathbb{R}^{m \times n}$ is called persistently exciting if there exist two strictly positive numbers $\mu$ and $T$ such that

$$
\int_{t}^{t+T} \phi(s) \phi(s)^{\top} d s \geq \mu I \quad \forall t \geq 0
$$

For certain systems appearing in the context of adaptive control and adaptive observers, this condition is known to be sufficient and necessary for the stability of the origin. More precisely, consider the following system:

$$
\begin{aligned}
& \dot{x}=A x+\mathcal{B}(t)^{\top} \theta \\
& \dot{\theta}=-\mathcal{B}(t) P x
\end{aligned}
$$

under the following conditions:

- the matrix $A$ is Hurwitz i.e., its eigen-values have all strictly negative real parts;

- the matrix $P$ is symmetric positive definite.

Then, the origin of the system is uniformly asymptotically stable; in particular, $\|x(t)\| \rightarrow 0$ as $t \rightarrow \infty$, if and only if $\mathcal{B}$ is persistently exciting. This result may be found in books on adaptive control ( $c f$. for instance [68]) and generalizations to the case when $A$ depends on system's trajectories appeared in [64].

Our main results on adaptive observers and their application in the master-slave synchronization problem rely on refinements of the result above for the cases when $A$ depend on the measurable outputs and $\mathcal{B}$ depends on the state. As we show, such formulations are adequate for a number of chaotic systems however, since we consider nonlinear systems one needs to start by posing properly the conditions of persistency of excitation along trajectories. We use a condition in the spirit of [64].

${ }^{3}$ Locally integrable is enough.

\section{B. On Observers}

With the previous discussions in mind, we are now ready to present a preliminary result on persistency-of-excitation based observer design for synchronization. A relatively simple observer for systems of the form (4) is given by

$$
\dot{\hat{x}}=A(y) \hat{x}-L(t, y) C(\hat{x}-x)
$$

where $L(\cdot, \cdot)$ is a design function chosen to satisfy the basic regularity assumption to ensure the well-posedness of the differential equation. The matrix function $L: \mathbb{R}_{\geq 0} \times \mathbb{R}^{m} \rightarrow \mathbb{R}^{n \times m}$ must be chosen in a way that the origin of the estimation error dynamics

$$
\dot{\bar{x}}=[A(y(t))-L(t, y(t)) C] \bar{x}, \quad \bar{x}:=\hat{x}-x
$$

be uniformly globally asymptotically stable.

Observers of the form (7) are reminiscent of Luenberger-type observers and are at the basis of designs for linear time-varying systems as for instance in [48], [49]. Note that here the differential (8) is time-varying and depends on the output trajectories $y(t)$. In the context of master-slave synchronization (8) represents the dynamics of the synchronization errors between that of the master's state $x$ and the slave's state $\hat{x}$. For example, a Lorenz oscillator, given by the equations:

$$
\begin{aligned}
& \dot{x}_{1}=\theta_{1}\left(x_{2}-x_{1}\right) \\
& \dot{x}_{2}=\theta_{2} x_{1}-x_{2}-x_{1} x_{3} \\
& \dot{x}_{3}=x_{1} x_{2}-\theta_{3} x_{3} .
\end{aligned}
$$

may be represented in the form (4) with

$$
y:=x_{1} ; \quad A(y):=\left[\begin{array}{ccc}
-\theta_{1} & \theta_{1} & 0 \\
\theta_{2} & -1 & -y \\
0 & y & -\theta_{3}
\end{array}\right]
$$

hence, the observability matrix for this system, from output $y=$ $x_{1}$ is

$$
\left[\begin{array}{c}
C \\
C A \\
C A^{2}
\end{array}\right]=\left[\begin{array}{ccc}
1 & 0 & 0 \\
-\theta_{1} & \theta_{1} & 0 \\
\theta_{1}\left(\theta_{1}+\theta_{2}\right) & -\theta_{1}\left(\theta_{1}+1\right) & -\theta_{1} y
\end{array}\right]
$$

which looses rank, for instance when $y=0$. Yet, it may be possible that the integral of this matrix along particular trajectories $y(t)$ over a window of length $T$ be of rank 3 for all $t$. In that case, the observer defined above, under an appropriate condition of persistency of excitation may reproduce the unmeasured states. More precisely we can establish the following.

Proposition 1: Consider System (8) and define $y_{t}:=y(t)$ for each $t$.

$$
\int_{t}^{t+T}\left[C^{\top} A(y(s))^{\top} C^{\top} \quad\left(A(y(s))^{2}\right)^{\top} C^{\top} \ldots\left(A(y(s))^{n-1}\right)^{\top} C^{\top}\right]^{\top} d s
$$


Assumption 1: Assume that there exists a globally uniformly bounded positive definite matrix function $P: \mathbb{R}_{\geq 0} \times \mathbb{R}^{m} \rightarrow$ $\mathbb{R}_{>0}^{n \times n}$ such that $p_{M} \geq\|P\|$ and, defining

$$
\begin{aligned}
\bar{A}\left(t, y_{t}\right):= & A\left(y_{t}\right)-L\left(t, y_{t}\right) C \\
-Q\left(t, y_{t}\right):= & \bar{A}\left(t, y_{t}\right)^{\top} P\left(t, y_{t}\right) \\
& +P\left(t, y_{t}\right)^{\top} \bar{A}\left(t, y_{t}\right)+\overparen{P\left(t, y_{t}\right)}
\end{aligned}
$$

we have the following for all $t \geq t_{\circ} \geq 0$ and all $x\left(t_{\circ}\right) \in \mathbb{R}^{n}$

1) $Q\left(t, y_{t}\right) \geq 0$

2) there exist $\mu$ and $T>0$ such that

$$
\int_{t}^{t+T} Q\left(\tau, y_{\tau}\right) d \tau \geq \mu I>0, \quad \forall t \geq 0 ;
$$

3) there exists $q_{M}>0$ such that $q_{M} \geq\left\|Q\left(t, y_{t}\right)\right\|$. Under these conditions the origin $\bar{x}=0$ of system (8) is uniformly globally exponentially stable (that is, uniformly exponentially stable for any initial states $\bar{x} \in \mathbb{R}^{n}$ and initial times $\left.t_{\circ} \geq 0\right)$.

The proof of Proposition 1 is provided in Appendix A. We draw the following immediate corollary.

Corollary 1: Consider two Lorenz systems: one master with state $x$ and one slave system with state $\hat{x}$ and assume that only the first variable of the master system is measurable. Synchronization is achieved under the conditions of Proposition 1. In particular, we have $\hat{x}(t) \rightarrow x(t)$ as $t \rightarrow \infty$.

Remark 1: In general, verifying Assumption 1 is hard since it must be done online; it is tantamount to verifying Assumption 2 below. However, it should be clear that points 1) and 2) of Assumption 1 hold if $Q$ is positive definite for all values of its arguments. Such assumption is common in related recent literature-cf. [7], [8], [47]-[49], [55]-[58] among others. To prove further on the arguments behind Assumption 1 we address the reader to [64].

Assumption 2: Let $\Phi_{x}\left(t, t_{\circ}\right)$ denote the transition matrix associated to $A\left(y_{t}\right)$, i.e., the solution of

$$
\left\{\begin{array}{l}
\dot{\Phi}_{x}\left(t, t_{\circ}\right)=A\left(y_{t}\right) \Phi_{x}\left(t, t_{\circ}\right), \\
\Phi_{x}\left(t_{\circ}, t_{\circ}\right)=I .
\end{array}\right.
$$

Assume that there exist positive numbers $T_{x}$ and $\mu_{x}$, such that, for all $t \geq 0$

$$
\int_{t}^{t+T_{x}} \Phi_{x}(\tau, t)^{\top} C^{\top} C \Phi_{x}(\tau, t) d \tau \geq \mu_{x} I .
$$

Remark 2: Note that condition (14) means that the output trajectory $y(t)$ is PE which is likely to hold if the system operates under a chaotic regime ${ }^{4}$. Yet we emphasize that PE is verifiable only on compact intervals of time as is naturally the case for any asymptotic property; moreover, since (14) is a condition along trajectories it may only be verified numerically and online.

Thus, Assumption 1 is little restrictive and allows to design efficient observers such as the so-called high-gain observers-cf. [35], [51] and references therein. Below, we give explicit formulae to compute $L$ and $P$ using a dynamic system. The following equations are reminiscent of designs for linear

\footnotetext{
${ }^{4}$ While this seems a reasonable conjecture, verified for a number of particular cases in simulations, we are not aware of a rigorous mathematical proof.
}

time-varying systems—cf. [47], [49] and for nonlinear systems in the so-called observable form-cf. [50], [62]. The fundamental difference here is that conditions are clearly stated along measured trajectories; note that they impose uniformity with respect to initial conditions. Such uniformity is fundamental for properties such as robustness with respect to "small disturbances"- $c f$. [66]. Hence, only by considering the trajectories explicitly one may lay the appropriate conditions for stability and robustness.

Proposition 2: Let Assumption 2 hold. For any given $\rho_{x}>0$, we define the observer gain for each trajectory, $L\left(t, y_{t}\right)$, as

$$
\begin{aligned}
L\left(t, y_{t}\right):= & P\left(t, y_{t}\right)^{-1} C^{\top} \\
\dot{P}\left(t, y_{t}\right)= & 2 C^{\top} C-\rho_{x} P\left(t, y_{t}\right)-P\left(t, y_{t}\right) A\left(y_{t}\right) \\
& -A\left(y_{t}\right)^{\top} P\left(t, y_{t}\right), \quad \forall t \geq t_{\circ}+T_{x} \\
P\left(t, y_{t_{\circ}}\right)= & P_{\circ}=P_{\circ}^{\top}>0 \quad \forall t \in\left[t_{\circ}, t_{\circ}+T_{x}\right] .
\end{aligned}
$$

Then, one has

$$
P\left(t, y_{t}\right) \geq \mu_{x} e^{-\rho_{x} T_{x}} I \text { for all } t \geq t_{\circ}+T_{x}
$$

and, on the other hand, the matrix $Q\left(t, y_{t}\right)$ from (11) with $P$ and $L$ given by (15) and (16), satisfies $Q\left(t, y_{t}\right) \equiv \rho_{x} P\left(t, y_{t}\right)$ so Assumption 1 holds.

The proof of the first part of this proposition is provided in Appendix B; the second part follows by direct calculation.

\section{Example}

We wrap up this section with some simulation results for the Lorenz system (9), using the observer from Proposition 2. In the simulation setup we assume that only the variable $x_{1}$ from the master is measurable and the observer (slave system) must reconstruct $x_{2}$ and $x_{3}$. In the simulation we have set $T_{x}=1 \mathrm{~s}$ and

$$
P\left(t_{\circ}\right)=\left[\begin{array}{lll}
5 & 1 & 2 \\
1 & 8 & 3 \\
2 & 3 & 9
\end{array}\right]
$$

whose eigenvalues are $\lambda_{1}=12.2006, \lambda_{2}=5.6517$ and $\lambda_{3}=$ 4.1477 .

The physical parameters $\theta_{1}, \theta_{2}$ and $\theta_{3}$ are chosen to make the Lorenz system describe a chaotic behavior i.e., $\theta_{1}=16$, $\theta_{2}=45.6$ and $\theta_{3}=4$. The initial states of the master system are set to one while those of the slave system (observer) are set to zero.

In view of Remark 2 and the facts that here $y=x_{1}$ and the Lorenz system operates in chaotic mode, it is expectable that the system satisfies the excitation condition imposed by Assumption 2.

In Fig. 1 we show the plots of the norms of the synchronization errors. One may appreciate the exponential decay of the errors to zero. We stress that this is done with the observer from Proposition 2 with measurement of $x_{1}$ only but with known parameters $\theta_{1}, \theta_{2}$ and $\theta_{3}$.

\section{AdAPtive ObSERVERS With Persistency of Excitation}

In the previous section we assumed that the constant parameters of system (4) are known. In practice this is an unrealistic assumption; for instance, in the case of a chaotic oscillator such 


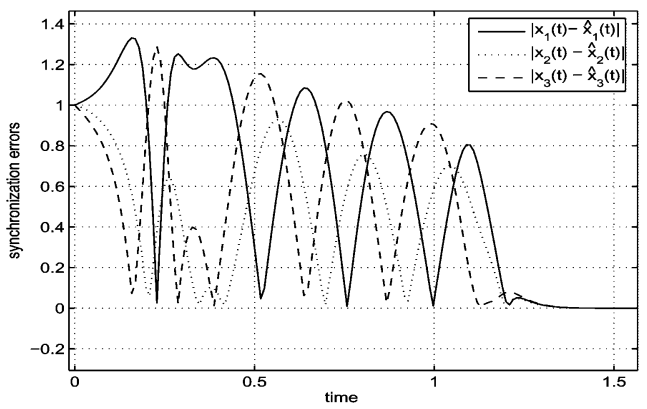

Fig. 1. Absolute value of synchronization errors for Lorenz system: $x_{i}$-states of master system and $\hat{x}_{i}$-states of slave system.

as the Lorenz system which may be realized as an electrical circuit—cf. [9], it is clear that one typically will dispose of approximate values of the parameters $\theta_{1}, \theta_{2}$ and $\theta_{3}$ which depend on the values of the physical components of the circuit. In such a case, it is desirable to design an observer with an adaptation law that updates the estimates $\hat{\theta}_{1}(t), \hat{\theta}_{2}(t)$ and $\hat{\theta}_{3}(t)$.

Hence, in a more general context we assume next that the system (4) may be written as

$$
\dot{x}=A(y) x+\Psi(x) \theta
$$

where the matrix $A$ depends only on the output $y \in \mathbb{R}^{m}$ (as opposed to the matrix in (10) where the system parameters are involved since they are considered to be known) and the matrix $\Psi$ depends only on the state but not on the unknown parameters. We assume that $\theta \in \Theta$ is a vector of unknown constant parameters and $\Theta$ is a compact of $\mathbb{R}^{m}$, that is, we separate the terms that depend on the unknown parameters from those terms that do not and we assume that the dependence of the system's dynamics on the unknown parameters $\theta$ is linear.

The model (20) covers or intersects with other interesting classes of systems studied in the literature of adaptive observers design. For instance, in [50] the systems that are considered (after a coordinate transformation) are such that $\theta$ appears only in dynamic equations of measured states. In [12] the class of systems considered restricts to the case where $A$ is constant or satisfies some structural conditions of relative degree, minimum phaseness - cf. [34], etc. This class covers systems with similar structure but where $A$ and $\Psi$ depend on time only and not on the state; that is, the case of linear time-varying systems. Thus, while restrictive from a strict systems' viewpoint the model (20) covers many chaotic oscillators studied in focussed articles such as on the Lorenz system -see the references cited in the Introduction.

We now make the following hypothesis on $\Psi$ :

Assumption 3: Let $x_{1}(t)$ and $x_{2}(t)$ be two solutions satisfying (20) for certain initial conditions. We assume that the function $\Psi$ along the trajectories $x_{1}(t)$ and $x_{2}(t)$ satisfies, for any vectors $\zeta \in \mathbb{R}^{m}$ with $\|\zeta\|=1$ and a positive constant $\psi_{M}>0,\left\|\left[\Psi\left(x_{1}(t)\right)-\Psi\left(x_{2}(t)\right)\right] \zeta\right\| \leq \psi_{M}\left\|x_{1}(t)-x_{2}(t)\right\|$ for all $t \geq t_{\circ} \geq 0$. We also assume that there exists $\psi_{0} \geq 0$ such that

$$
\max _{\|\zeta\|=1}\left\|\zeta^{\top} \Psi(0) \zeta\right\| \leq \psi_{0}
$$

The first part of the previous assumption may be satisfied, in particular, in the following cases which make sense for chaotic systems:

- the matrix function $\Psi(\cdot)$ is once continuously differentiable and the trajectories $x(t)$ are bounded for all $t$;

- the function $\Psi(\cdot)$ is globally Lipschitz: $\|\left[\Psi\left(x_{1}\right)\right.$ $\left.\Psi\left(x_{2}\right)\right] \zeta\left\|\leq \psi_{M}\right\| x_{1}-x_{2} \|$ for all $x_{1}, x_{2} \in \mathbb{R}^{n}$.

The second sufficient condition for Assumption 3, that is, globally Lipschitz, is restrictive in the context of general nonlinear systems. The second alternative assumption, i.e., boundedness on $x(t)$ is not restrictive in the present context of synchronization if we recall that $x(t)$ corresponds to the solutions of an ordinary differential equation $\dot{x}=f(t, x, \theta)$ such that for a particular choice of $\theta$ the system exhibits a chaotic behavior and therefore, $x(t)$ is bounded. Boundedness of $\dot{x}(t)$ follows directly from the regularity hypotheses imposed on $f$ to guarantee existence and uniqueness of solutions. On the other hand, that the condition in the first bullet above is sufficient for Assumption 3 to hold, follows by invoking the Mean Value theorem for multivariable functions-cf. [69]. Thus, Assumption 3 is not restrictive in the present context of master-slave synchronization.

Under these conditions, an adaptive observer for systems of the form (20) is given by

$$
\dot{\hat{x}}=A(y) \hat{x}-L(t, y) C(\hat{x}-x)+\Psi(\hat{x}) \hat{\theta}
$$

where $L(\cdot, \cdot)$ satisfies the basic regularity assumption and the persistency of excitation condition implicitly defined in Assumption 1. Using (20), and defining $\bar{x}:=\hat{x}-x, \bar{\theta}:=\hat{\theta}-\theta$ the estimation error dynamics is given by

$$
\begin{aligned}
\dot{\bar{x}}= & {[A(y)-L(t, y) C] \bar{x}+\Psi(\bar{x}+x(t)) \bar{\theta} } \\
& +\Phi(t, \bar{x}, x(t), \theta) \\
\Phi(t, \bar{x}, x(t), \theta):= & {[\Psi(\bar{x}+x(t))-\Psi(x(t))] \theta . }
\end{aligned}
$$

Assumption 3 and the assumption that $\theta \in \Theta$ where $\Theta$ is a compact of appropriate dimension imply that there exists $\theta_{M}>$ 0 such that

$$
\|\Phi(t, \bar{x}(t), x(t), \theta)\| \leq \psi_{M} \theta_{M}\|\bar{x}(t)\|=: \phi_{M}\|\bar{x}(t)\| .
$$

Next, consider the adaptation law

$$
\dot{\hat{\theta}}(t)=-\gamma \Psi(\hat{x}(t))^{\top} P\left(t, y_{t}\right) \hat{x}(t), \quad \gamma>0
$$

which, considering that $\dot{\theta}=0$, is equivalent to

$$
\begin{aligned}
& \dot{\bar{\theta}}=-\gamma \Psi(\bar{x}+x(t))^{\top} P\left(t, y_{t}\right) \bar{x} \\
&-\gamma \Psi(\bar{x}+x(t))^{\top} P\left(t, y_{t}\right) x(t)
\end{aligned}
$$

with $\gamma>0$. We also impose a persistency-of-excitation condition on the function $\Psi(x(t))$ :

Assumption 4: The function $\Psi(x(t))$ is such that there exist positive numbers $\mu_{\psi}$ and $T_{\psi}$ such that, for any unitary vector $\zeta \in \mathbb{R}^{m}$

$$
\int_{t}^{t+T_{\psi}}\|\Psi(x(\tau)) \zeta\| d \tau \geq \mu_{\psi}, \quad \forall t \geq 0
$$




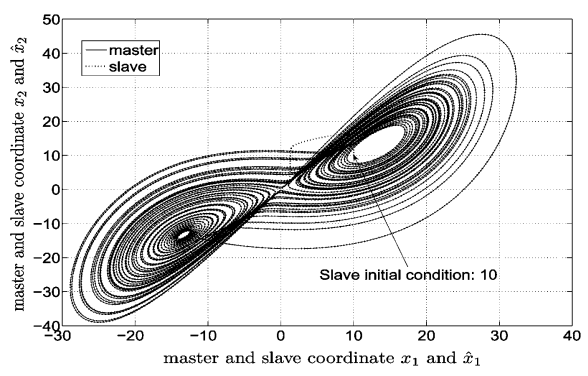

Fig. 2. Lorenz slave and master systems: phase portrait of $x_{1}$ versus $x_{2}$ (master, continuous line) and $\hat{x}_{1}$ versus $\hat{x}_{2}$ (slave -dotted line).

Assumption 4 is a structural condition on the function $\Psi(\cdot)$ as well as on the richness of the trajectories $x(t)$.

Under these conditions we have the following.

Proposition 3: The origin of the estimation error dynamics corresponding to $\bar{x}$ and $\bar{\theta}$, i.e., (23) and (26), is uniformly semi-globally practically asymptotically stable provided that Assumptions 1, 3 and 4 are satisfied and the solutions $x(t)$ and their derivatives $\dot{x}(t)$ are bounded for all $t$.

Roughly, Proposition 3 establishes conditions for the state and parameter estimation errors to converge to a small neighborhood of the origin. In the context of master-slave synchronization of chaotic systems, Proposition 3 establishes conditions under which two chaotic systems with unknown constant parameters, synchronize, in the event that only an output of the master system is measurable.

\section{A. Example: Synchronization of Two Lorenz Oscillators}

Let us consider the synchronization problem for two Lorenz systems $-c f$. (9). We assume to measure $y_{1}=x_{1}$ and that $\theta_{3}$ is unknown. Under such conditions the system can be rewritten in the form (20) with $y=x_{1}$

$$
A(y):=\left[\begin{array}{ccc}
-\theta_{1} & \theta_{1} & 0 \\
\theta_{2} & -1 & -y_{1} \\
0 & y_{1} & 0
\end{array}\right], \quad \Psi(x):=\left[\begin{array}{ccc}
0 & 0 & 0 \\
0 & 0 & 0 \\
0 & 0 & -x_{3}
\end{array}\right]_{(28} .
$$

The functions above satisfy the basic regularity conditions and $\Psi$ is globally Lipschitz.

We note that not any choice of measurable output leads to a realizable synchronization algorithm since the detectability assumption may not be verified. For instance, if only $x_{1}$ is measured and all parameters are unknown we have

$$
\begin{aligned}
& A(y):=\left[\begin{array}{ccc}
0 & 0 & 0 \\
0 & -1 & -y_{1} \\
0 & y_{1} & 0
\end{array}\right] \\
& \Psi(x):=\left[\begin{array}{ccc}
x_{2}-x_{1} & 0 & 0 \\
0 & x_{1} & 0 \\
0 & 0 & -x_{3}
\end{array}\right] .
\end{aligned}
$$

The observability matrix for this system becomes

$$
\left[\begin{array}{c}
C \\
C A \\
C A^{2}
\end{array}\right]=\left[\begin{array}{lll}
1 & 0 & 0 \\
0 & 0 & 0 \\
0 & 0 & 0
\end{array}\right]
$$

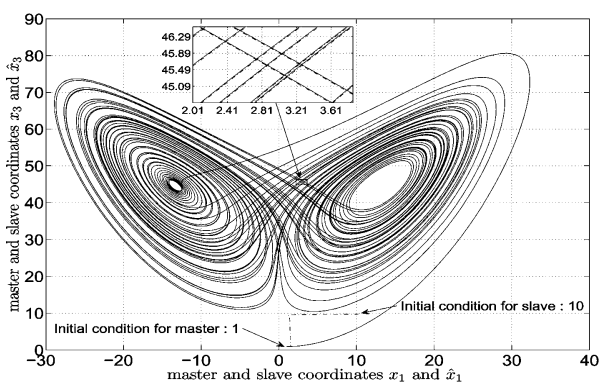

Fig. 3. Lorenz slave and master systems: phase portrait of $x_{1}$ versus $x_{3}$ (master, continuous line) and $\hat{x}_{1}$ versus $\hat{x}_{3}$ (slave $\cdots$ dotted line).

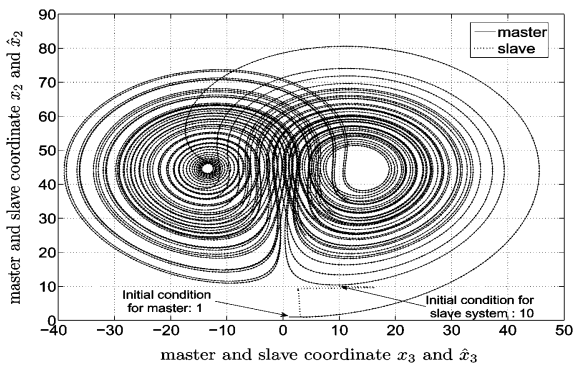

Fig. 4. Lorenz slave and master systems: phase portrait of $x_{2}$ versus $x_{3}$ (master, continuous line) and $\hat{x}_{2}$ versus $\hat{x}_{3}$ (slave $\cdots$ dotted line).

which is always of rank 1 . In other words, it is impossible to design a state and parameter estimator of the type (22) by measuring only $x_{1}$ and having no knowledge of the parameters 5 .

We tested the proposed algorithm in simulation under the following conditions. For a chaotic behavior we set the parameters of the master system to $\theta_{1}=16, \theta_{2}=45.6$ and $\theta_{3}=4$; the master initial states are set to $x(0)=[1 ; 1 ; 1]$; the slave initial states are set to $\hat{x}(0)=[10 ; 10 ; 10]$ and the initial estimate of the master's parameter $\theta_{3}$ to $\hat{\theta}_{3}(0)=2$. The observer parameters are set to $\rho_{x}=1000, T_{x}=0.01 \mathrm{~s}$ and $P_{\mathrm{o}}=I$. The adaptation gain is set to $\gamma=2$.

The simulation results are presented in Figs. 2-6. The phase portraits of the master and slave systems are depicted in Figs. 2-4; one can appreciate the good match between master and slave trajectories. In Fig. 5 we present the graphs of the three synchronization errors against time. The estimated value of the parameter $\theta_{3}$ is depicted in Fig. 6 where it is appreciated a small mismatch between the true $\left(\theta_{3}=4\right)$ and estimated value $\left(\hat{\theta}_{3}=4.16\right)$. This small error is expectable: Proposition 3 establishes practical asymptotic stability in the case of parametric uncertainty. Indeed, notice that, in the ideal case that $\bar{x} \equiv 0$ after a time say $t^{*}$, we have from (25) $\dot{\bar{\theta}}=-\gamma \Psi(x(t))^{\top} P\left(t, y_{t}\right) x(t)$ where $x(t)$ 'has a chaotic behavior'. On the other hand, note that the 'stable' term $-\rho_{x} P$ in (16) drives the solution of the latter to zero (in the absence of the other terms) hence, it may be inferred that the matrix $P$ becomes considerably small. Moreover, since $x(t)$ is chaotic it is also bounded so $\dot{\bar{\theta}} \approx 0$ even though $\bar{\theta}$ does not necessarily tends to zero asymptotically. As established by the proposition one can only guarantee practical asymptotic stability.

\footnotetext{
${ }^{5} \mathrm{We}$ are not aware of any result for such case by any other method either.
} 


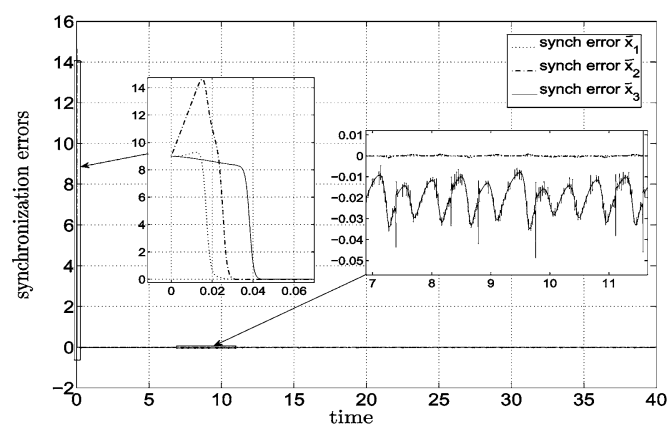

Fig. 5. Lorenz slave and master systems: synchronization errors for the three variables i.e., $\bar{x}_{1}, \bar{x}_{2}$ and $\bar{x}_{3}$.

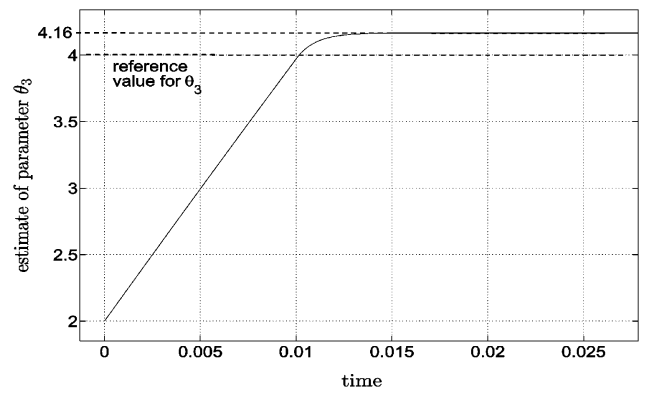

Fig. 6. Lorenz slave and master systems: Estimate of parameter $\theta_{3}$. Value for master system $\theta_{3}=4$; estimated value $\hat{\theta}_{3}=4.1661$.

\section{RobUSt AdAPTIVE SYNCHRONIZATION VIA PE OBSERVERS}

We consider now, systems with additional nonlinearities $B(t, x)$ that depend only on the state, time and known parameters, i.e., systems of the form

$$
\dot{x}=A(y) x+\Psi(x) \theta+B(t, x)
$$

where $B$ satisfies the same assumptions as $\Psi$, uniformly in $t$ :

Assumption 5: Let $r>0$ be arbitrarily fixed and let $\xi(t)$ and $\xi^{\prime}(t)$ be two solutions satisfying (30) for respective initial conditions $\xi_{\circ}$ and $\xi_{\circ}^{\prime}$ such that $\max \left\{\left\|\xi_{\circ}\right\|,\left\|\xi_{\circ}^{\prime}\right\|\right\} \leq r$. We assume that there exists $b_{M}$ such that the function $B$, along the trajectories $\xi(t)$ and $\xi^{\prime}(t)$ satisfies

$$
\left\|B(t, \xi(t))-B\left(t, \xi^{\prime}(t)\right)\right\| \leq b_{M}\left\|\xi(t)-\xi^{\prime}(t)\right\|
$$

for all $t \geq t_{\circ} \geq 0$ and all initial states such that $\max \left\{\left\|\xi_{\circ}\right\|,\left\|\xi_{\circ}^{\prime}\right\|\right\} \leq r$.

This condition on $B$ holds under similar conditions as for $\Psi-c f$. Assumption 3. We stress that $B(t, \cdot)$ needs not to be globally Lipschitz. In this respect it is convenient to stress that the trajectories of chaotic systems are bounded.

For the system (30) the observer equation becomes

$$
\dot{\hat{x}}=A(y) \hat{x}-L(t, y) C(\hat{x}-x)+\Psi(\hat{x}) \hat{\theta}+B(t, \hat{x})
$$

and the estimation (synchronization) error dynamics is

$$
\dot{\bar{x}}=[A(y)-L(t, y) C] \bar{x}+\Psi(\bar{x}+x(t)) \bar{\theta}+\Phi(t, \bar{x}, x(t), \theta)
$$

where

$$
\begin{aligned}
\Phi(t, \bar{x}, x(t), \theta):=[\Psi(\bar{x}+x(t)) & -\Psi(x(t))] \theta \\
& +B(t, \bar{x}+x(t))-B(t, x(t)) .
\end{aligned}
$$

Assumptions 3 and 5 together with the hypothesis that $\theta \in \Theta$ where $\Theta$ is a compact of appropriate dimension imply that there exists $\theta_{M}>0$ such that

$$
\begin{aligned}
\|\Phi(t, \bar{x}(t), x(t), \theta)\| & \leq \psi_{M} \theta_{M}\|\bar{x}(t)\|+b_{M}\|\bar{x}(t)\| \\
& =: \phi_{M}\|\bar{x}(t)\|
\end{aligned}
$$

which, considering (24), redefines $\phi_{M}$. This makes it apparent that there is no loss of generality in considering that $B$ does not contain any unknown parameters since those terms may be embedded in $\Psi(x)$. As a matter of fact, a direct corollary from Proposition 3 follows for systems (30); that is, the adaptive observer from previous section still ensures the property of semi-global practical asymptotic stability.

Corollary 2: The origin of the estimation error dynamics corresponding to $\bar{x}$ and $\bar{\theta}$, i.e., (33) and (26), is uniformly semi-globally practically asymptotically stable provided that Assumptions 1, 3, 4 and 5 are satisfied and the solutions $x(t)$ and their derivatives $\dot{x}(t)$ are bounded for all $t$.

In the context of synchronization this is tantamount to ensuring master-slave synchronization with a small error. This is stated in the following proposition which contains all the previous results.

Proposition 4: Consider a chaotic master system of the form (30) where $\theta$ is such that the solutions $x(t)$ exhibit a chaotic behavior. Let $y=C x$ be a measurable output of the master system. Construct a slave system according to the dynamics (32), (25). Then, under the conditions of Corollary 2 a slave system synchronizes with the master; in particular, $\hat{x}(t)$ approaches $x(t)$ as $t \rightarrow \infty$. Moreover:

1) in the case that the parameters $\theta$ are unknown, the errors $\|x(t)-\hat{x}(t)\|$ and $\|\theta-\hat{\theta}\|$ approach a small neighborhood of the origin as $t \rightarrow \infty$. Moreover, the size of this neighborhood may be reduced by increasing the persistency of excitation, i.e., $\mu_{x}$ and $\mu$;

2 ) in the case that the parameters $\theta$ are unknown but $C=I$, i.e. the whole master system's state is measurable, perfect synchronization occurs and the parameters $\theta$ may be estimated if the persistency of excitation condition (27) holds;

3 ) in the case that the parameters $\theta$ are known, the slave system will achieve perfect synchronization provided that the persistency of excitation condition imposed in Assumption 1 holds.

The statement in point 1) generalizes previous results which rely either on a Lipschitz condition on the additional nonlinearities $B$ or, on positivity of the matrix $Q$ in Assumption 1 or both $-c f$. Remark 1 and references mentioned there; the statement in point 2) is reminiscent of controlled synchronization under parameter uncertainty when the whole state is measurable—cf.e.g., [32], [70], [71] and references in the latter; the statement in point 3 ) generalizes results on observer-based synchronization, as briefly treated in Section III-cf. e.g., [7], [35], etc.

\section{A. Example: the Chaotic Oscillator of Lü-Cf. [72]}

We consider as example the chaotic oscillator from [72]

$$
\begin{aligned}
& \dot{x}_{1}=\theta_{1} x_{1}-x_{2} x_{3}+\theta_{4} \\
& \dot{x}_{2}=\theta_{2} x_{2}+x_{1} x_{3} \\
& \dot{x}_{3}=\theta_{3} x_{3}+x_{1} x_{2}
\end{aligned}
$$




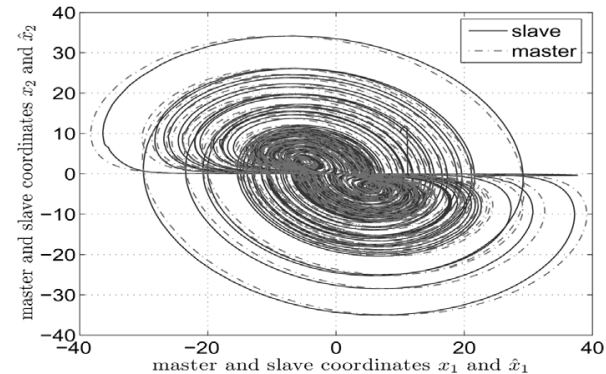

Fig. 7. Lü et al. chaotic oscillator: phase portraits of the master system- $x_{1}$ versus $x_{2}$ and for the slave system $-\hat{x}_{1}$ versus $\hat{x}_{2}$.

where $\theta_{1}=-\theta_{2} \theta_{3} / \theta_{2}+\theta_{3}$. In the simulation setup we use the high-gain adaptive observer (32), (15) with measurable master's states $x_{2}$ and $x_{3}$. We also assume that $\theta_{3}$ and $\theta_{4}$ are unknown. We consider the term $x_{1} x_{2}$ as a "neglected" dynamics i.e., we define $B(t, x):=\left[\begin{array}{lll}0 & 0 & x_{1} x_{2}\end{array}\right]^{\top}$. This term satisfies the Lipschitz hypothesis on $B$ along trajectories, in view of the boundedness of $x_{1}(t)$ under a chaotic regime: indeed, for any $r$ and any initial states satisfying $\left\|x_{0}\right\| \leq r$ there exists $R$ such that $\|x(t)\| \leq R$ for all $t \geq t_{\circ} \geq 0$. Hence, condition (31) holds with $b_{M}=2 R$. To see this we observe that $\left\|B(t, \xi(t))-B\left(t, \xi^{\prime}(t)\right)\right\|=\left|\xi_{1}(t) \xi_{2}(t)-\xi_{1}^{\prime}(t) \xi_{2}^{\prime}(t)\right| \leq$ $\left|\xi_{1}(t)\right|\left|\xi_{2}(t)-\xi_{2}^{\prime}(t)\right|+\left|\xi_{2}^{\prime}(t)\right|\left|\xi_{1}(t)-\xi_{1}^{\prime}(t)\right| \leq 2 R\left\|\xi(t)-\xi^{\prime}(t)\right\|$. However, it is clear that $B(t, x)$ does not satisfy sector or Lipschitz conditions—cf. e.g., [7], [8], [55], [57], [58] since it is a bilinear function of the states.

According to [20] this oscillator presents chaotic behavior for parameter values $\theta_{2}=-10, \theta_{3}=-4$ and $\left|\theta_{4}\right|<19.2$ and initial states $x(0)=[3 ;-4 ; 2]^{\top}$; we set $\theta_{4}=0$; with these values $\theta_{1}=-2.2587$. The observer design parameters are set to $\gamma=0.025$ and $\rho_{x}=1000$ and $T_{x}=0.1$. The slave system's initial states are set to $\hat{x}_{1}(0)=10, \hat{x}_{2}(0)=10, \hat{x}_{3}(0)=10$. The matrix $P$ is initialized at $P_{\circ}=I$.

The simulation results are shown in Figs. 7-11. Figs. 7 and 8 show the phase portraits of the slave system's variables $\hat{x}_{2}$ and $\hat{x}_{3}$ relative to the estimated state $\hat{x}_{1}$; on the same figures we present for comparison, the corresponding phase portraits for the master system. For a better appreciation the small synchronization error $\hat{x}_{1}-x_{1}$ against time, is depicted in Fig. 9. Fig. 10 shows the estimated parameters $\hat{\theta}_{1}$ and $\hat{\theta}_{2}$; indeed, even though $\theta_{1}$ is a function of $\theta_{2}$ and $\theta_{3}$ which is known, $\hat{\theta}_{1}$ and $\hat{\theta}_{2}$ are computed according to the adaptation law (25) as independent parameters. In this figure we show the small estimation error for $\hat{\theta}_{1}$ relative to $\theta_{1}$ albeit a more important mismatch between $\hat{\theta}_{2}$ and $\theta_{2}$. Finally, some plots representative of the evolution of the observer gain $L$ against time, are presented in Fig. 11: in the simulation, the measurable output is $y=\left[\begin{array}{ll}x_{2} & x_{3}\end{array}\right]^{\top}$ so we write $y=C x$ with $C=\operatorname{diag}\left[\begin{array}{lll}0 & 1 & 1\end{array}\right]$. The figure shows the evolution of the three eigenvalues of the matrix $L\left(t, y_{t}\right):=$ $P^{-1}\left(t, y_{t}\right) C^{T}$ against time. While the gains' magnitudes may be a drawback of high-gain observers for implementation purposes, it is worth to emphasize again that this observer is only one case for which Assumption 1 holds. We recall at this point that, roughly, it is needed an observer guaranteeing asymptotic stability of the system $\dot{\bar{x}}=[A(y)-L(t, y) C] \bar{x}$, along the trajectories $y(t)$; a sufficient but not necessary condition for this is the more restrictive hypothesis (used in a number of references-cf.

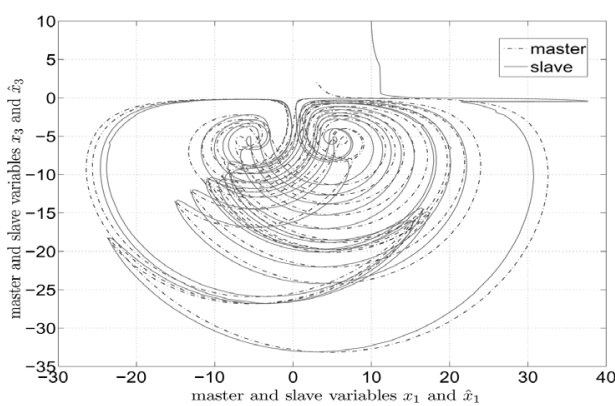

Fig. 8. Lü et al. chaotic oscillator: phase portraits of the master system- $x_{1}$ versus $x_{3}$ and for the slave system $-\hat{x}_{1}$ versus $\hat{x}_{3}$.

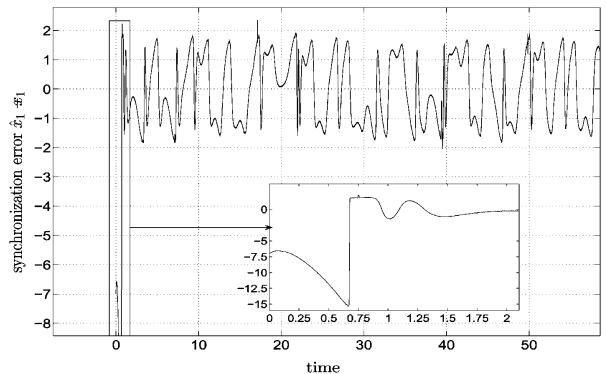

Fig. 9. Lü et al. chaotic oscillator: synchronization error i.e., difference between master's variable $x_{1}$ and slave's variable $\hat{x}_{1}$.
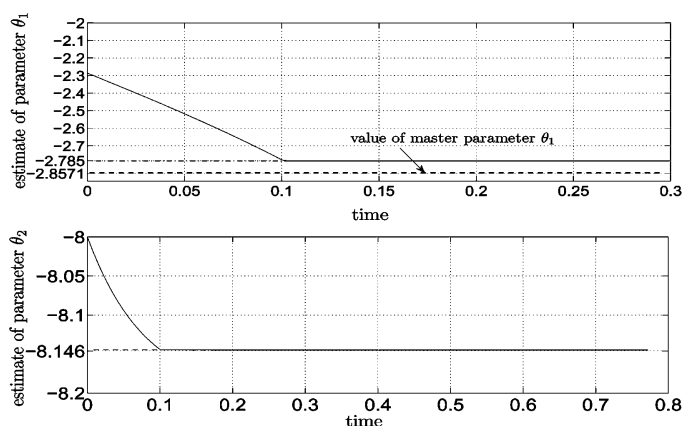

Fig. 10. Lü et al. chaotic oscillator: estimates of parameters $\theta_{1}$ and $\theta_{2}$.



Fig. 11. Lü et al. chaotic oscillator: three eigenvalues $\lambda_{1}\left(L\left(t, y_{t}\right)\right)$, $\lambda_{2}\left(L\left(t, y_{t}\right)\right), \lambda_{3}\left(L\left(t, y_{t}\right)\right)$ of the observer gain $L\left(t, y_{t}\right)$ on different scales.

Remark 1) that the matrix $Q$ in the assumption is positive definite along trajectories, uniformly for all initial conditions.

\section{CONCLUSION}

We presented an adaptive observer scheme for systems' chaotic synchronization. Our approach applies to a class of 
systems with dynamics that contains, essentially, three terms: the first is assumed to depend linearly in the unmeasured variables and have no parameter uncertainty, the second is assumed to depend nonlinearly in the unmeasured states and linearly in the uncertain constant parameters, a third term is considered to depend both on time and (un)measured states without parametric uncertainty. Considering all three terms together, the class of systems is fairly wide, covering many classical examples of chaotic oscillators. Our conditions are stated in terms of persistency of excitation which is a mild condition for convergence in the context of time-varying systems and covers many other results on observer-based synchronization previously published. For illustration, we have addressed two examples of master-slave synchronization, in particular, for the Lorenz and for the Lü oscillators.

We believe that connections with some of the mentioned works on swithching-topology networks ${ }^{6}$ may be established in terms of persistency of excitation which is clearly linked to the notion of average. Indeed, PE has been used formally to establish stability among a networks of systems with time-varying $(\mathrm{PE})$ interconnections in [73]. Future research is aimed in this direction.

\section{APPENDIX \\ PROOFS}

Proof of Proposition 1: Consider Assumption 1. It is a standard result in adaptive control literature $-c f$. [33] that (12) is equivalent to

$$
\int_{t}^{t+T} \xi^{\top} Q\left(\tau, y_{\tau}\right) \xi d \tau \geq \mu \quad \forall t \geq 0
$$

for any unitary vector $\xi \in \mathbb{R}^{n}$. That is, $\phi(t):=\xi^{\top} Q\left(t, y_{t}\right) \xi$ is PE and satisfies $\phi_{M} \geq\|\phi(t)\|$ for all $t \geq 0$. Consider now the function $V_{1}(t, \bar{x}):=\bar{x}^{\top} P\left(t, y_{t}\right) \bar{x}$; its total derivative along the solutions of $\dot{\bar{x}}=\bar{A}\left(y_{t}\right) \bar{x}$ yields, by assumption, $\dot{V}_{1}=-\bar{x}^{\top} Q\left(t, y_{t}\right) \bar{x} \leq 0$. This implies that, defining $p_{m}$ and $p_{M}$ as

$$
p_{m}:=\inf _{\substack{\|\xi\|=1 \\ t \geq 0}} \xi^{\top} P\left(t, y_{t}\right) \xi \quad p_{M}:=\sup _{\substack{\|\xi\|=1 \\ t \geq 0}} \xi^{\top} P\left(t, y_{t}\right) \xi,
$$

the solutions of $\dot{\bar{x}}=\bar{A}\left(y_{t}\right) \bar{x}$ satisfy

$$
\|\bar{x}(t+T)\|^{2} \leq \frac{p_{M}}{p_{m}}\|\bar{x}(\tau)\|^{2}, \quad \forall \tau \in[t, t+T], t \geq 0 .
$$

It follows from this, the equation $\dot{V}_{1}(\tau, \bar{x}(\tau))=$ $-\bar{x}(\tau)^{\top} Q\left(\tau, y_{\tau}\right) \bar{x}(\tau)$ and (36) that

$$
\begin{aligned}
& V_{1}(t, \bar{x}(t))-V_{1}(t+T, \bar{x}(t+T)) \\
& \quad \geq \int_{t}^{t+T} \bar{x}(\tau)^{\top} Q\left(\tau, y_{\tau}\right) \bar{x}(\tau) d \tau \\
& \quad \geq \int_{t}^{t+T}\|\bar{x}(\tau)\|^{2}\left(\inf _{\substack{\|\xi\|=1 \\
\tau \geq 0}} \xi^{\top} Q\left(\tau, y_{\tau}\right) \xi\right) d \tau
\end{aligned}
$$

${ }^{6}$ Many references have been left out due to space constraints; the reader is invited to see other work on this topic, particularly by the independent authors Belykh, Bollt and di Bernardo.

$$
\begin{aligned}
& \geq \int_{t}^{t+T} \frac{p_{m}}{p_{M}}\left(\inf _{\substack{\|\xi\|=1 \\
\tau \geq 0}} \xi^{\top} Q\left(\tau, y_{\tau}\right) \xi\right) d \tau\|\bar{x}(t+T)\|^{2} \\
& \geq \frac{\mu p_{m}}{p_{M}}\|\bar{x}(t+T)\|^{2}
\end{aligned}
$$

which, integrating on both sides from $t_{0}$ to $\infty$, implies that

$$
\begin{aligned}
\int_{t_{0}}^{t_{0}+T} \frac{p_{m} \mu}{p_{M}}\|\bar{x}(t)\|^{2} d t+\int_{t_{0}}^{t_{0}+T} & V_{1}(t, \bar{x}(t)) d t \\
& \geq \int_{t_{0}}^{\infty} \frac{p_{m} \mu}{p_{M}}\|\bar{x}(t)\|^{2} d t
\end{aligned}
$$

hence

$$
\left(T \frac{p_{m}}{p_{M}}+\frac{2 p_{m}}{\mu}\right)\left\|\bar{x}\left(t_{0}\right)\right\|^{2} \geq \int_{t_{0}}^{\infty}\|\bar{x}(t)\|^{2} d t .
$$

It follows from [64, Lemma 3] that the origin of $\dot{\bar{x}}=A\left(y_{t}\right) \bar{x}$ is globally exponentially stable, uniformly in $y_{t}$. Moreover, defining

$$
c:=\sqrt{\max \left\{\left(T \frac{p_{m}}{p_{M}}+\frac{2 p_{m}}{\mu}\right), \frac{p_{m}}{p_{M}}\right\}}
$$

we have

$$
\|\bar{x}(t)\| \leq 2 c e^{1 / 2}\left\|\bar{x}_{\circ}\right\| \mathrm{e}^{-1 / 2 c^{2}\left(t-t_{\circ}\right)} .
$$

Proof of Proposition 2: Positiveness of $p\left(t, y_{t}\right)$ : In the sequel we drop the arguments: we write $P$ for $P\left(t, y_{t}\right), A$ for $A\left(t, y_{t}\right)$ and $\Phi_{x}$ for $\Phi_{x}\left(t, t_{\circ}\right)$.

Multiplying by $e^{\rho_{x} t}$ on both sides of (16) we obtain

$$
\frac{d}{d t}\left(e^{\rho_{x} t} P\right)=-e^{\rho_{x} t}\left(A^{\top} P+P A-2 C^{\top} C\right) .
$$

Let $\Phi_{x}$ denote the transition matrix defined in Assumption 2. Then, left and right-multiplying on both sides of (43) by $\Phi_{x}^{\top}$ and $\Phi_{x}$ respectively, we obtain

$$
\Phi_{x}^{\top} \stackrel{\cdot}{e^{\rho_{x} t} P} \Phi_{x}=-e^{\rho_{x} t} \Phi_{x}^{\top}\left(A^{\top} P+P A-2 C^{\top} C\right) \Phi_{x} .
$$

Next, we recall from (13) that

$$
\Phi_{x}^{\top} A^{\top} P=\dot{\Phi}_{x}^{\top} P
$$

hence

$$
P A \Phi_{x}=P \dot{\Phi}_{x}
$$

therefore

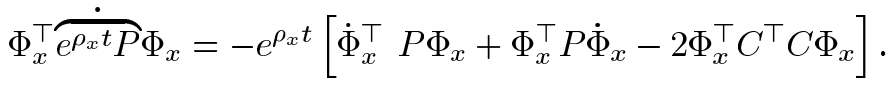

On the other hand,

$$
\begin{aligned}
& \frac{d}{d t}\left(e^{\rho_{x} t} \Phi_{x}^{\top} P \Phi_{x}\right) \\
& =e^{\rho_{x} t}\left[\Phi_{x}^{\top}(\dot{P}+\rho P) \Phi_{x}+\dot{\Phi}_{x}^{\top} P \Phi_{x}+\Phi_{x}^{\top} P \dot{\Phi}_{x}\right] .
\end{aligned}
$$

Using (16) and (44) in the latter, we obtain

$$
\frac{d}{d t}\left(e^{\rho_{x} t} \Phi_{x}^{\top} P \Phi_{x}\right)=2 e^{\rho_{x} t} \Phi_{x}^{\top} C^{\top} C \Phi_{x} .
$$


Integrating the previous expression from $t_{\circ}$ to $t+T_{x}$ and recalling that $P_{\circ}=P\left(t_{0}, y_{t_{\circ}}\right)>0$, we get

$$
\begin{aligned}
e^{\rho_{x}\left(t+T_{x}\right)} & \Phi_{x}\left(t+T_{x}, t_{\circ}\right)^{\top} P\left(t+T_{x}\right) \Phi_{x}\left(t+T_{x}, t_{\circ}\right) \\
& \geq \int_{t_{\circ}}^{t+T_{x}} 2 e^{\rho_{x} \tau} \Phi_{x}\left(\tau, t_{\circ}\right)^{\top} C(\tau)^{\top} C(\tau) \Phi_{x}\left(\tau, t_{\circ}\right) d \tau .
\end{aligned}
$$

Multiplying on both sides by $e^{-\rho_{x}\left(t+T_{x}\right)}$ and, on the left by $\Phi_{x}\left(t_{\circ}, t+T_{x}\right)^{\top}$ and on the right by $\Phi_{x}\left(t_{\circ}, t+T_{x}\right)$, we obtain

$$
\begin{aligned}
P\left(t+T_{x},\right. & \left.y_{t+T_{x}}\right) \geq \int_{t_{\circ}}^{t+T_{x}} 2 e^{\rho_{x}\left(\tau-t-T_{x}\right)} \Phi_{x}\left(t_{\circ}, t+T_{x}\right)^{\top} \\
& \times \Phi_{x}\left(\tau, t_{\circ}\right)^{\top} C(\tau)^{\top} C(\tau) \Phi_{x}\left(\tau, t_{\circ}\right) \Phi_{x}\left(t_{\circ}, t+T_{x}\right) d \tau .
\end{aligned}
$$

Reducing the size of the window of integration and using the transitivity of $\Phi_{x}$, we obtain

$$
\begin{array}{r}
P\left(t+T_{x}, y_{t+T_{x}}\right) \geq \int_{t}^{t+T_{x}} 2 e^{\rho_{x}\left(\tau-t-T_{x}\right)} \Phi_{x}\left(\tau, t+T_{x}\right)^{\top} \\
\times C(\tau)^{\top} C(\tau) \Phi_{x}\left(\tau, t+T_{x}\right) d \tau .
\end{array}
$$

Hence, since

$$
\tau \geq t \quad \Rightarrow \quad \tau-t-T_{x} \geq-T_{x}
$$

we get

$$
\begin{aligned}
P\left(t+T_{x}, y_{t+T_{x}}\right) \geq e^{-\rho_{x} T_{x}} \int_{t}^{t+T_{x}} 2 & \Phi_{x}\left(\tau, t+T_{x}\right)^{\top} C(\tau)^{\top} \\
& \times C(\tau) \Phi_{x}\left(\tau, t+T_{x}\right) d \tau,
\end{aligned}
$$

and consequently, from (14),

$$
P\left(t, y_{t}\right) \geq \mu_{x} e^{-\rho_{x} T_{x}} I, \quad \forall t \geq t_{\circ}+T_{x} .
$$

Proof of Proposition 3: The dynamics of the estimation errors $z:=\operatorname{col}[\bar{x}, \bar{\theta}]$ is now given by

$$
\begin{aligned}
\dot{z} & =F(t, z) z+K(t, z) \\
F(t, z) & :=\left[\begin{array}{cc}
{\left[A\left(y_{t}\right)-L\left(t, y_{t}\right) C\right]} & -\gamma \Psi(\bar{x}+x(t)) \\
-\gamma \Psi(\bar{x}+x(t))^{\top} P\left(t, y_{t}\right) & 0
\end{array}\right] \\
K(t, z) & :=\left[\begin{array}{c}
\Phi(t, \bar{x}, x(t), \theta) \\
-\gamma \Psi(\bar{x}+x(t))^{\top} P\left(t, y_{t}\right) x(t)
\end{array}\right]
\end{aligned}
$$

where $\Phi$ is defined by (23b). Let $\beta_{x}$ be such that $\|x(t)\| \leq \beta_{x}$ for all $t \geq 0$ then, from (24) and (21), it follows that

$$
\begin{gathered}
\|K(t, z)\| \leq\left(\psi_{M} p_{M} \beta_{x}+\phi_{M}\right)\left\|z_{1}\right\|+p_{M} \psi_{0} \beta_{x} \\
=: b_{1}\left\|z_{1}\right\|+b_{2} .
\end{gathered}
$$

Consider now the following claim to be true (the proof is provided farther below).

Claim 1: Under the conditions of Proposition 1 the origin of $\dot{z}=F(t, z) z$ is UGAS and uniformly exponentially stable on any ball.
From the proof of Claim $1-c f$. Section C.1 we have, for any $r \geq 0$ and $t_{\circ} \geq 0$,

$$
\left\|z\left(t_{\circ}\right)\right\|<r \quad \Rightarrow \quad\|z(t)\| \leq k\left\|z\left(t_{\circ}\right)\right\| \mathrm{e}^{-\gamma\left(t-t_{\circ}\right)}
$$

where $k:=2 c e^{1 / 2}, \gamma:=1 / 2 c^{2}$ and $c>0$ is defined below (55). It follows, from the proof of [65, Th. 4.14], that there exists $V_{4}: \mathbb{R}_{\geq 0} \times B_{R} \rightarrow \mathbb{R}_{\geq 0}$ with $R:=k r$, such that

$$
\begin{aligned}
\left(\frac{1-\mathrm{e}^{-2 q_{M} T}}{2 q_{M}}\right)\|z\|^{2} & \leq V_{4}(t, z) \leq\left(\frac{1-\mathrm{e}^{-2 \gamma T}}{2 \gamma}\right)\|z\|^{2} \\
\frac{\partial V_{4}}{\partial t}+\frac{\partial V_{4}}{\partial z} F(t, z) z & \leq-\left(1-e^{-2 \gamma T}\right)\|z\|^{2} \\
\left\|\frac{\partial V_{4}}{\partial z}\right\| & \leq \frac{2}{\gamma-q_{M}}\left[1-\mathrm{e}^{-\left(\gamma-q_{M}\right) T}\right] .
\end{aligned}
$$

Evaluating the time derivative of $V_{4}(t, z)$ along the trajectories of (46a) and using (47) we obtain

$$
\begin{aligned}
& \dot{V}_{4}(t, z) \leq-\left(1-e^{-2 \gamma T}\right)\|z\|^{2} \\
&+\frac{2}{\gamma-q_{M}}\left[1-\mathrm{e}^{-\left(\gamma-q_{M}\right) T}\right]\left(b_{1}\|z\|^{2}+b_{2}\|z\|\right)
\end{aligned}
$$

hence if, for any given $\epsilon>0, b_{1}, b_{2}$ and $z$ satisfy

$$
\begin{gathered}
b_{1} \leq \frac{\left(1-\mathrm{e}^{-2 \gamma T}-\epsilon\right)\left(\gamma-q_{M}\right)}{4\left[1-\mathrm{e}^{-\left(\gamma-q_{M}\right) T}\right]} \\
\|z\| \geq b_{2} \frac{4\left[1-\mathrm{e}^{-\left(\gamma-q_{M}\right) T}\right]}{\left(1-\mathrm{e}^{-2 \gamma T}-\epsilon\right)\left(\gamma-q_{M}\right)}
\end{gathered}
$$

we obtain

$$
\dot{V}_{4}(t, z) \leq-\epsilon\|z\|^{2} .
$$

It follows that the solutions are uniformly ultimately bounded-cf. [65, p. 172] for all initial conditions such that $\left\|z_{\mathrm{o}}\right\|<r$. On the other hand, the term on the right-hand side of (50) may be reduced at will by enlarging $\gamma$ (i.e., by enlarging $c$ hence, $\mu$ and $\mu_{\psi}$ ) while the calculations above hold for $r$ arbitrarily large but finite; hence, it follows that the origin is semi-globally uniformly practically asymptotically stable.

Proof of Claim 1: The proof relies on the result from Proposition 1 and the following

Claim 2: There exists $c_{z 1}<\infty$ such that the function $t \mapsto z_{1}$ generated by the differential equations $\dot{z}=F(t, z) z$ where $F$ is defined in (46b), satisfies

$$
\int_{t_{\circ}}^{\infty}\left\|z_{1}(t)\right\| d t \leq c_{z 1}\left\|z_{\circ}\right\| \quad \forall t \geq t_{\circ} \geq 0
$$

and moreover, the origin of $\dot{z}=F(t, z) z$ is UGS with $\kappa(s):=$ $c_{z 0} s-c f$. Ineq. (2), and

$$
c_{z 0}:=\sqrt{\frac{\max \left\{p_{M}, \frac{1}{\gamma}\right\}}{\min \left\{p_{m}, \frac{1}{\gamma}\right\}}} .
$$


Claim 3: There exists $c_{z 2}<\infty$ such that the function $t \mapsto z_{2}$ generated by the differential equations $\dot{z}=F(t, z) z$ where $F$ is defined in (46b), satisfies

$$
\int_{t_{\circ}}^{\infty}\left\|z_{2}(t)\right\| d t \leq c_{z 2}\left\|z_{\circ}\right\| \quad \forall t \geq t_{\circ} \geq 0
$$

From Claims 2 and 3 above it follows that

$$
\int_{t_{\circ}}^{\infty}\|z(t)\| d t \leq c_{z}\left\|z_{\circ}\right\| \quad \forall t \geq t_{\circ} \geq 0
$$

where $c_{z}:=\max \left\{c_{z 1}, c_{z 2}\right\}$. It follows from [64, Lemma 3] that the origin is uniformly exponentially attractive on any ball, that is, it is uniformly globally attractive and, moreover, for any $r>0$ we have

$$
\left\|z\left(t_{\circ}\right)\right\|<r \quad \Rightarrow \quad\|z(t)\| \leq 2 c e^{1 / 2}\left\|z\left(t_{0}\right)\right\| \mathrm{e}^{-1 / 2 c^{2}\left(t-t_{0}\right)}
$$

with $c:=\max \left\{c_{z}, c_{z 0}\right\}$. We conclude that the origin of the system is UGAS and uniformly exponentially stable on any ball.

Notice that as $c$ decreases, the rate of convergence $\gamma:=$ $-1 / 2 c^{2}$ increases.

Proof of Claim 2: The proof follows naturally from the proof of Proposition 1. Consider the positive definite function

$$
V_{2}(t, z):=z_{1}^{\top} P\left(t, y_{t}\right) z_{1}+\frac{1}{\gamma}\left\|z_{2}\right\|^{2}
$$

its total derivative along the solutions of $\dot{z}=F(t, z) z$ yields $\dot{V}_{2}(t, z)=\dot{V}_{1}(t, z) \leq 0$ which implies that $p_{m}\|\bar{x}(t)\|^{2}+$ $(1 / \gamma)\|\bar{\theta}(t)\|^{2} \leq\|z(t)\|^{2} \leq p_{M}\left\|\bar{x}\left(t_{\circ}\right)\right\|^{2}+(1 / \gamma)\left\|\bar{\theta}\left(t_{\circ}\right)\right\|^{2}$. It follows that the system is UGS, in particular, it satisfies

$$
\|z(t)\| \leq c_{z 0}\left\|z\left(t_{\circ}\right)\right\| \quad \forall t \geq t_{\circ} \geq 0
$$

with $c_{z 0}$ as defined in (52). The first part of the claim follows observing that (40) still holds for the trajectories of $\dot{z}=F(t, z) z$ hence, (51) holds with

$$
c_{z 1}(\mu, T):=\sqrt{T \frac{p_{M}}{p_{m}}+\frac{2 p_{m}}{\mu}} .
$$

Notice that for each fixed $T, c(\mu, T) \rightarrow 0$ as $\mu \rightarrow \infty$.

Proof of Claim 3: Let $r>0$ be an arbitrary number and define $R:=c_{z 0} r$. Consider the system $\dot{z}=F(t, z) z$ with initial conditions satisfying $\left\|z_{\circ}\right\|<r$; then, we have $\|z(t)\|<R$ for all $t \geq t_{\circ}$. Consider the function $V_{3}: \mathbb{R}_{\geq 0} \times B_{R} \rightarrow \mathbb{R}_{\geq 0}$ defined as

$$
\begin{aligned}
& V_{3}(t, z)=V_{2}(t, z) \\
& \quad-\varepsilon\left(\int_{t}^{\infty} e^{(t-\tau)}\left\|\Psi(x(\tau)) z_{2}\right\|^{2} d \tau+z_{1}^{\top} \Psi(x(t)) z_{2}\right) .
\end{aligned}
$$

Under Assumption 4 we have

$$
-\int_{t}^{\infty} e^{(t-\tau)}\left\|\Psi(x(\tau)) z_{2}\right\|^{2} d \tau \leq-\mu_{\psi} e^{-T_{\psi}}\left\|z_{2}\right\|^{2}
$$

hence, in view of the boundedness of $x(t)$ and the Lipschitz property of $\Psi$ we have $V_{3}$ is positive definite for sufficiently small $\varepsilon$; moreover, there exist positive numbers $\alpha_{1}, \alpha_{2}$ such that

$$
\alpha_{1}\|z\|^{2} \leq V_{3}(t, z) \leq \alpha_{2}\|z\|^{2} .
$$

On the other hand, the time derivative of $V_{3}$ along the trajectories of $\dot{z}=F(t, z) z$ yields

$$
\begin{aligned}
& \dot{V}_{3}(t, z) \leq \dot{V}_{2}(t, z)-\varepsilon e^{-T_{\psi}} \frac{\mu_{\psi}^{2}}{T_{\psi}}\left\|z_{2}\right\|^{2} \\
& \left.-\varepsilon z_{1}^{\top} \overparen{\Psi(x(t))} z_{2}-\gamma \Psi(x(t)) P z_{1}\right] \\
& -\varepsilon\left[(A-L C) z_{1}+\left(\Psi\left(z_{1}+x(t)\right)-\Psi(x(t))\right) z_{2}\right] \\
& \times \Psi(x(t)) z_{2} \text {. }
\end{aligned}
$$

Under the regularity assumptions made on $x(t)$, $\Psi$ etc., and considering that $\|z(t)\|<R$, it follows that there exists a number $c_{R}$ such that

$$
\begin{aligned}
\dot{V}_{3}(t, z(t)) \leq & -\varepsilon e^{-T_{\psi}} \frac{\mu_{\psi}^{2}}{T_{\psi}}\left\|z_{2}(t)\right\|^{2} \\
& +\varepsilon c_{R}\left[\left\|z_{1}(t)\right\| z_{2}(t)+\left\|z_{2}(t)\right\|\right] \\
\leq & -\left(\varepsilon e^{-T_{\psi}} \frac{\mu_{\psi}^{2}}{T_{\psi}}-\frac{\varepsilon}{2}\right)\left\|z_{2}(t)\right\|^{2} \\
& +\left(c_{R}^{2}+1\right)\left\|z_{1}(t)\right\|^{2}
\end{aligned}
$$

which, defining $c_{\theta}:=\left(\varepsilon e^{-T_{\psi}} \mu_{\psi}^{2} / T_{\psi}-\varepsilon / 2\right)$ is equivalent to

$$
\begin{aligned}
\int_{t_{\circ}}^{\infty} c_{\theta}\left\|z_{2}(t)\right\|^{2} d t \leq & V_{3}\left(t_{\circ}, z\left(t_{\circ}\right)\right) \\
& +\left(c_{R}^{2}+1\right) c_{z_{1}}^{2} \int_{t_{\circ}}^{\infty}\left\|z_{1}(t)\right\|^{2} d t
\end{aligned}
$$

The result follows with

$$
c_{z 2}\left(T_{\psi}, \mu_{\psi}\right):=\sqrt{\frac{\alpha_{2}+\left(c_{R}^{2}+1\right) c_{z_{1}}^{2}}{c_{\theta}}} .
$$

Notice that $c_{z 2}\left(T_{\psi}, \mu_{\psi}, \mu\right) \rightarrow 0$ as $\mu_{\psi} \rightarrow \infty$ and $\mu_{\psi} \rightarrow \infty$.

Proof of Proposition 4: The proof follows from the developments of the previous section. In the first case, the synchronization error dynamics is given exactly by (23) and (26) whose origin has been showed to be uniformly semi-globally practically asymptotically stable. In the second case, the synchronization error dynamics corresponds to (23) and, instead of (26),

$$
\dot{\bar{\theta}}=-\gamma \Psi(\bar{x}+x(t))^{\top} P\left(t, y_{t}\right) \bar{x} \quad \gamma>0 .
$$

In this case, $b_{2}$ in (47b) is zero and therefore, the calculations involved in the proof of Proposition 3 hold for all $\|z\| \geq 0$. In the case of the high-gain observer, notice that the synchronization may be achieved from any initial errors. In the third case, the synchronization dynamics is given simply by (23) with $\bar{\theta} \equiv 0$ and the result follows from the proof of Proposition 1 for sufficiently large $\mu$. 


\section{ACKNOWLEDGMENT}

The authors are grateful to the anonymous reviewers for their many constructive remarks and for pointing out several relevant references on synchronization in the context of networks of oscillators.

\section{REFERENCES}

[1] I. I. Blekhman, Synchronization in Science and Technology. New York: ASME Press, 1988.

[2] L. M. Pecora and T. L. Carroll, "Synchronization in chaotic systems," Phys. Rev. A, vol. 64, pp. 821-824, 1990.

[3] G. Chen and X. Dong, From Chaos to Order: Methodologies, Perspectives, and Applications. Singapore: World Scientic, 1998.

[4] A. L. Fradkov, H. Nijmeijer, and A. Markov, "Adaptive observer-based synchronisation for communications," Int. J. Bifurc. Chaos, vol. 10, no. 12 , pp. 2807-2814, 2000 .

[5] K. M. Cuomo, A. V. Oppenheim, and S. H. Strogatz, "Synchronization of Lorenz-based chaotic circuits with applications to communications," IEEE Trans. Circuits Syst. I, Fundam. Theory Appl., vol. 40, no. 10, pp. 626-633, Oct. 1993.

[6] M. Boutayeb, M. Darouach, and H. Rafaralahy, "Generalized statespace observers for chaotic synchronization and secure communication," IEEE Trans. Circuits Syst. I, Fundam. Theory Appl., vol. 49, no. 3, pp. 345-349, Mar. 2002.

[7] J. Amirazodi, E. E. Yaz, A. Azemi, and Y. I. Yaz, "Nonlinear observer performance in chaotic synchronization with application to secure communication," in Proc. Int. Conf. Control Appl., Glasgow, Scotland, 2002, pp. 76-81.

[8] F. Zhu, "Observer-based synchronization of uncertain chaotic system and its application to secure communications," Chaos, Solitons Fractals vol. 40, no. 5, pp. 2384-2391, Jun. 2009.

[9] Z. He, K. Li, L. Yuang, and Y. Sui, "A robust digital structure communications scheme based on sporadic chaos synchronization," IEEE Trans. Circuits Syst. I, Reg. Papers, vol. 47, no. 3, pp. 397-403, Mar. 2000.

[10] N. Balmforth, C. Tresser, P. Worfolk, and W. C. Wu, "Master-slave synchronization and the lorenz equations," Chaos, vol. 7, no. 3, pp. 392-394, 1997.

[11] G. Malescio, "Synchronization of the lorenz system through continuous feedback control," Phys. Rev. E, vol. 53, pp. 6566-6568, 1996.

[12] B. R. Andrievskii, V. O. Nikifarov, and A. L. Fradkov, "Adaptive observer-based synchronization of the nonlinear nonpassifiable systems," Automat. Remote Contr., vol. 68, no. 7, pp. 1186-1200, 2007.

[13] I. I. Blekman, A. L. Fradkov, H. Nijmeijer, and A. Y. Pogromsky, "On self-synchronization and controlled synchronization," Syst. Contr. Lett., vol. 31, pp. 299-305, 1997.

[14] I. Boutat-Baddas, J. P. Barbot, D. Boutat, and R. Tauleigne, "Sliding mode observers and observability in chaotic synchronization," Math. Problems Eng., no. 1, pp. 11-31, 2004.

[15] C. Zhou and C.-H. Lai, "Decoding information by following parametric modulation with parametric adaptive contol," Phys. Rev. E, vol. 59, no. 6, pp. 2269-6636, 1999

[16] U. Parlitz, "Estimating model parameters from time series by autosynchronization," Phys. Rev. Lett, vol. 76, pp. 1232-1235, 1996.

[17] L. Li, H. Peng, X. Wang, and Y. Yang, "Comment on two papers of chaotic synchronization," Phys. Lett. A, vol. 333, pp. 269-270, 2004.

[18] W. Perruquetti and J. P. Barbot, Chaos in Automatic Control. Boca Raton, FL: CRC Press, 2005.

[19] H. Nijmeijer and A. Rodríguez-Angeles, Synchronization of Mechanical Systems. Singapore: World Scientific, 2003, vol. 46, Nonlinear Science, Series A.

[20] J. H. Park, "Chaos synchronization of a chaotic system via nonlinear control," Chaos, Solitons Fractals, vol. 25, pp. 579-584, 2005.

[21] H. H. Chen, "Chaos control and global synchronization of liu chaotic systems using linear balanced feedback control," Chaos, Solitons Fractals, vol. 40, no. 1, pp. 466-473, Apr. 2007.

[22] Z.-M. Ge and T.-N. Lin, "Chaos, chaos control and synchronization of electro-mechanical gtrostat system," J. Sound Vibrat., vol. 259, no. 3, pp. 585-603, 2003.

[23] I. M. Kypriandis and I. N. Stouboulos, "Chaotic synchronization of three coupled oscillators with ring connection," Chaos, Solitons Fractals, vol. 17, pp. 327-336, 2003.

[24] M. Porfiri and M. d. Bernardo, "Criteria for global pinning-controllability of complex networks," Automatica, no. 12, pp. 3100-3106, 2008.
[25] M. Hasler and I. Belykh, "Blinking long-range connections increase the functionality of locally connected networks," IEICE Trans. Fundam. vol. 88-A, no. 10, pp. 2647-2655, 2005.

[26] I. Belykh, V. Belykh, and M. Hasler, "Blinking model and synchronization in small-world networks with a time-varying coupling," Phys. $D$, vol. 195, no. 1-2, pp. 188-206, 2004.

[27] D. J. Stilwell, E. M. Bollt, and D. G. Roberson, "Sufficient conditions for fast switching synchronization in time varying network topologies," SIAM J. Dyn. Syst., vol. 5, no. 1, pp. 140-156, 2006.

[28] F. Sorrentino, M. d. Bernardo, and F. Garofalo, "Synchronizability and synchronization dynamics of complex networks with degree-degree mixing," Int. J. Bifurcat. Chaos, vol. 17, no. 7, pp. 2419-2434, 2007.

[29] Z. Li and G. Chen, "Global synchronization and asymptotic stability of complex dynamical networks," IEEE Trans. Circuits Syst. II, Exp. Briefs, vol. 53, no. 1, pp. 28-33, Jan. 2006.

[30] M. Porfiri, D. J. Stilwell, and E. M. Bollt, "Synchronization in random weighted directed networks," IEEE Trans. Circuits Syst. I, Reg. Papers, vol. 55, no. 10, pp. 3170-3177, Nov. 2008.

[31] D. Yu and A. Wu, "Comment on: Estimating model parameters from time series by autosynchronization," Phys. Rev. Lett., vol. 94, 2005 219401.

[32] A. Loría and A. Zavala, "Adaptive tracking control of chaotic systems with applications to synchronization," IEEE Trans. Circuits Syst. I, Reg. Papers, vol. 54, no. 9, pp. 2019-2030, Sep. 2007.

[33] K. S. Narendra and A. M. Annaswamy, Stable Adaptive Systems. Englewood Cliffs, NJ: Prentice-Hall, 1989.

[34] P. Ioannou and J. Sun, Robust Adaptive Control. Englewood Cliffs, NJ: Prentice-Hall, 1996.

[35] H. Nijmeijer and I. Mareels, "An observer looks at synchronization," IEEE Trans. Circuits Syst. I, Fundam. Theory Appl., vol. 44, no. 10, pp. 882-890, Oct. 1997.

[36] T. Kailath, Linear Systems. Englewood Cliffs, NJ: Prentice Hall, 1980.

[37] C.-T. Chen, Linear System Theory and Design, 3rd ed. Oxford, U.K. Oxford Univ. Press, 1998.

[38] R. Marino and P. Tomei, "Global adaptive output feedback control of nonlinear systems-Part I: Linear parameterization," IEEE Trans. Autom. Contr. vol. 38, no. 1, pp. 17-32, Jan. 1993.

[39] A. L. Fradkov and A. Y. Pogromsky, "Speed gradient control of chaotic continuous-time systems," IEEE Trans. Circuits Syst. I, Fundam. Theory Appl., vol. 43, no. 11, pp. 907-914, Nov. 1996.

[40] A. M. Harb and B. A. Harb, "Controlling chaos in Josephson-junction using nonlinear backstepping controller," IEEE Trans. Appl. Supercond., vol. 16, no. 4, pp. 1988-1998, Dec. 2006.

[41] E. O. C. Grebogi and J. A. Yorke, "Controlling chaos," Phys. Rev. Lett, vol. 64, pp. 1196-1199, 1990.

[42] T. Kapitaniak, Controlling Chaos. New York: Academic, 1996

[43] E. Cherrier, M. Boutayeb, and J. Ragot, "Observers-based synchronization and input recovery for a class of nonlinear chaotic models," IEEE Trans. Circuits Syst. I, Reg. Papers, vol. 53, no. 9, pp. 1977-1988, Sep. 2006.

[44] A. L. Fradkov, B. Andrievsky, and R. J. Evans, "Adaptive observerbased synchronization of chaotic systems with first-order coder in the presence of information constraints," IEEE Trans. Circuits Syst. I, Reg. Papers, vol. 55, no. 6, pp. 1685-1694, Jul. 2008.

[45] S. Wang, H. Luo, C. Yue, and X. Liao, "Parameter identification of chaos system based on unknown parameter observer," Phys. Lett. A, vol. 372, pp. 2603-2607, 2007.

[46] Z. Jiang, "Advanced feedback control of chaotic systems," IEEE Trans. Circuits Syst. I, Fundam. Theory Appl., vol. 49, no. 2, pp. 244-249, 2002.

[47] Q. Zhang, "Adaptive observers for MIMO linear time-varying systems," IEEE Trans. Autom. Contr., vol. 47, no. 3, pp. 525-529, Mar. 2002.

[48] Q. Zhang and B. Deylon, "A new approach to adaptive observer design for MIMO systems," in Proc. IEEE Amer. Contr. Conf., Arlington, VA, 2001, pp. 1545-1550.

[49] Q. Zhang, "Revisiting different adaptive observers through a unified formulation," in Proc. 44th. IEEE Conf. Decision Contr., Seville, Spain, 2005, pp. 3067-3072.

[50] G. Besancon, "Remarks on nonlinear adaptive observer design," Syst. Contr. Lett., vol. 41, pp. 270-280, 2000

[51] H. Hammouri and J. d. L. Morales, "On systems equivalence and observer synthesis," in Proc. Conf. New Trends Syst. Theory, Genoa/Italy, 1991, pp. 341-347. 
[52] J. Gauthier, H. Hammouri, and S. Othman, "A simple observer for nonlinear systems -application to bioreactors," IEEE Trans. Autom. Contr., vol. 37, no. 6, pp. 875-880, Jun. 1992.

[53] G. Besançon, A Viewpoint on Observability and Observer Design for Nonlinear Systems, H. Nijemeijer and T. I. Fossen, Eds. London, U.K.: Springer Verlag, 1999, Lecture Notes in Control and Information Sciences, ch. New directions in nonlinear observer design.

[54] G. Besançon, "Contributions à l'étude et à l'observation des systèmes non linéaires avec recours au calcul formel," Ph.D. dissertation, INPG, France, 1996.

[55] D. Ya-Li and M. Sheng-Wei, "Adaptive observer for a class of nonlinear systems," Acta Automat. Sinica, vol. 33, no. 10, pp. 1081-1084, 2007.

[56] R. Aguilar-López and R. Martínez-Guerra, "Synchronization of a class of chaotic signals via robust observer design," Chaos, Solitons Fractals, vol. 37, pp. 581-587, 2008.

[57] M. Chen and W. Min, "Unknown-input observer based chaotic secure communication," Phys. Lett. A, vol. 372, pp. 1595-1600, 2008.

[58] F. Zhu, "Full order and reduced-order observer-based synchronization for chaotic systems with unknown disturbances," Phys. Lett. A, vol. 372, pp. 223-232, 2008.

[59] J.-G. Lu and D. Hill, "Global asymptotical synchronization of chaotic lur'e systems using sampled data: A linear matrix inequality approach," IEEE Trans. Circuits Syst. II, Exp. Briefs, vol. 55, no. 6, pp. 586-590, Jun. 2008.

[60] Q.-L. Han, "On designing time-varying delay feedback controllers for master-slave synchronization of Lur'e systems," IEEE Trans. Circuits Syst. I, Reg. Papers, vol. 54, no. 7, pp. 1573-1583, Jul. 2007.

[61] J. L. J. Cao and D. W. C. Ho, "Adaptive stabilization and synchronization for chaotic Lur'e systems with time-varying delay," IEEE Trans. Circuits Syst. I, Reg. Papers, vol. 55, no. 5, pp. 1347-1356, May 2008.

[62] G. Besançon and J. de L. Morales, "On adaptive observers for state affine systems and application to synchronous machines," presented at the 42st. IEEE Conf. Decision Contr., Maui, HI, Dec. 9-12, 2003, Paper 689.

[63] A. Loría and J. de Leon Morales, "On persistently exciting observers and a nonlinear separation principle: Application to a generator," Int. J. Contr., vol. 76, no. 6, pp. 607-618, 2003.

[64] A. Loría and E. Panteley, "Uniform exponential stability of linear timevarying systems: Revisited," Syst. Contr. Lett., vol. 47, no. 1, pp. 13-24, 2002.

[65] H. Khalil, Nonlinear Systems, 3rd ed. Englewood Cliffs, NJ: Prentice-Hall, 2002.

[66] A. Loría and E. Panteley, Advanced Topics in Control Systems Theory, A. Loría, F. Lamnabhi-Lagarrigue, and E. Panteley, Eds. London, U.K.: Springer Verlag, 2006, vol. Lecture Notes in Control and Information Sciences, ch. Stability, told by its developpers, pp. 1-84628$313-2$.

[67] A. Chaillet and A. Loría, "Uniform semiglobal practical asymptotic stability for non-autonomous cascaded systems and applications," $\mathrm{Au}$ tomatica, vol. 44, no. 2, pp. 337-347, 2008.

[68] B. D. O. Anderson, R. Bitmead, C. Johnson, Jr, P. Kokotović, R. Kosut, I. Mareels, L. Praly, and B. Riedle, Stability of Adaptive Systems. Cambridge, MA: MIT Press, 1986.

[69] A. E. Taylor and W. R. Mann, Advanced Calculus. New York: Wiley, 1983.

[70] Y. P. Tian and X. Yu, "Adaptive control of chaotic dynamical systems using invariant manifold approach," IEEE Trans. Circuits Syst. I, Fundam. Theory Appl., vol. 47, no. 10, pp. 1537-1542, Oct. 2000.

[71] A. L. Fradkov and A. Y. Markov, "Adaptive synchronization of chaotic systems based on speed gradient method and passification," IEEE Trans. Circuits Syst. I, Fundam. Theory Appl., vol. 44, no. 10, pp. 905-912, Oct. 1997.

[72] J. Lü, G. Chen, and D. Cheng, "A new chaotic system and beyond: The generalized Lorenz-like system," Int. J. Bifurc. Chaos, vol. 14, no. 5, pp. 1507-1537, 2004.
[73] A. R. Teel, E. Panteley, D. Popovic, and A. Loría, "Matrosov's theorem using a family of auxiliary functions: An analysis tool to aid time-varying nonlinear control design,” 2003 [Online]. Available: http://front.math.ucdavis.edu/math.OC/0304255

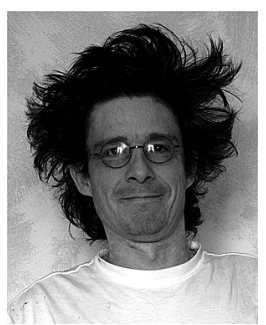

Antonio Loria (S'90-M'96) was born in Mexico City, Mexico, in 1969. He received the B.Sc. degree in electronic engineering from the Instituto Tecnológico y de Estudios Superiores de Monterrey (ITESM), Monterrey, Mexico, in 1991 and the M.Sc. and Ph.D. degrees in control engineering from the UTC, Compiègne, France, in 1993 and 1996, respectively.

From December 1996 through December 1998, he was an Associate Researcher at the University of Twente, Enschede, The Netherlands; Norwegian University of Science and Technology (NTNU), Norway, and the CCEC of the University California at Santa Barbara. He is the "Directeur de Recherche" (Senior Researcher), at the French National Centre of Scientific Research (CNRS). He is with the "Laboratoire de Signaux et Systemes", SUPELEC, since December 2002. His research interests include control systems theory and practice, electrical systems, analysis and control of chaos.

Dr. Loria serves as Associate Editor for Systems and Control Letters, Automatica, IEEE TRANSACTIONS ON AUTOMATIC CONTROL, , IEEE TRANSACTIONS ON CONTROL SYSTEMS TECHNOLOGY, and is member of the IEEE Control Systems Society (CSS) Conference Editorial Board.

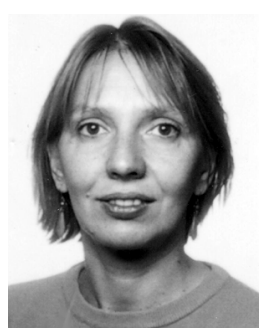

Elena Panteley was born in Leningrad, USSR. She received the M.Sc. and Ph.D. degrees in applied mathematics from the State University of St. Petersburg, Russia.

She is an Associate Researcher (CR1) of the French National Centre of Scientific Research (CNRS), at Laboratoire de Signaux et Systemes. From 1986 to 1998, she held a research position with the Institute for Problem of Mechanical Engineering of the Academy of Science of Russia, St. Petersburg. During 1998, she was an Associate Researcher at the Center for Control Engineering and Computation of the University of California at Santa Barbara. During 1999 she was with the INRIA Rhone Alpes, Monbonnot, France. She is coauthor of more than 90 scientific articles and book chapters. Her research interests are stability of nonlinear time-varying systems, control of electromechanical systems, nonlinear and robust control.

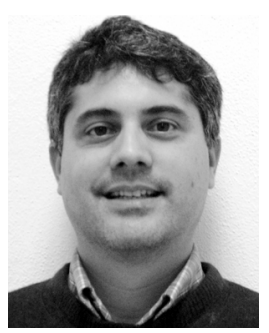

Arturo Zavala-Río was born in Merida, Yucatan, Mexico, in 1967. He received the B.Sc. degree in electronic systems engineering and the M.Sc. degree in control engineering from the Instituto Tecnologico y de Estudios Superiores de Monterrey (ITESM), Mexico, in 1989 and 1992 respectively, and the D.E.A. (Diplome d'Études Aprofondies) and $\mathrm{Ph} . \mathrm{D}$. degrees in automatic control from the Institut National Politechnique de Grenoble, France, in 1994 and 1997, respectively.

He held a Visiting Research position at the Mechanical Engineering Laboratory, Japan, from January to December 1998, and at the Université de Tehcnologie de Compiegne, France, from September 2001 to August 2002. Since August 2001, he holds a Researcher position at the Instituto Potosino de Investigacion Cientifica y Tecnologica, Mexico. His research interests include the modelling, analysis, and control of nonlinear systems. 